\title{
Is There a Geometric Module for Spatial Orientation? Insights From a Rodent Navigation Model
}

\author{
Denis Sheynikhovich, Ricardo Chavarriaga, Thomas Strösslin, Angelo Arleo, and Wulfram Gerstner \\ École Polytechnique Fédérale de Lausanne
}

\begin{abstract}
Modern psychological theories of spatial cognition postulate the existence of a geometric module for reorientation. This concept is derived from experimental data showing that in rectangular arenas with distinct landmarks in the corners, disoriented rats often make diagonal errors, suggesting their preference for the geometric (arena shape) over the nongeometric (landmarks) cues. Moreover, sensitivity of hippocampal cell firing to changes in the environment layout was taken in support of the geometric module hypothesis. Using a computational model of rat navigation, the authors proposed and tested the alternative hypothesis that the influence of spatial geometry on both behavioral and neuronal levels can be explained by the properties of visual features that constitute local views of the environment. Their modeling results suggest that the pattern of diagonal errors observed in reorientation tasks can be understood by the analysis of sensory information processing that underlies the navigation strategy employed to solve the task. In particular, 2 navigation strategies were considered: (a) a place-based locale strategy that relies on a model of grid and place cells and (b) a stimulus-response taxon strategy that involves direct association of local views with action choices. The authors showed that the application of the 2 strategies in the reorientation tasks results in different patterns of diagonal errors, consistent with behavioral data. These results argue against the geometric module hypothesis by providing a simpler and biologically more plausible explanation for the related experimental data. Moreover, the same model also describes behavioral results in different types of water-maze tasks.
\end{abstract}

Keywords: geometric module, view matching, navigational strategies, computational model, grid cells

Supplemental materials: http://dx.doi.org/10.1037/a0016170.supp

Throughout the history of research on animal learning, there have been conflicting views concerning the fundamental issue of what animals learn during training in a spatial task. Cognitive theorists such as Tolman (1948) proposed that animals acquire knowledge of the environment layout, or a cognitive map, whereas other theorists proposed that animal learning consists of formation of stimulus-response (S-R) habits (Hull, 1943). Recent behavioral and lesion data suggest that animals are able to use both the map-based and S-R navigational strategies when solving spatial tasks; these strategies are mediated by distinct memory systems (O'Keefe \& Nadel, 1978; Packard \& McGaugh, 1992, 1996; White $\&$ McDonald, 2002) and hence may be learned in parallel and compete for control of behavior (Devan \& White, 1999; Packard \& McGaugh, 1996; White \& McDonald, 2002).

Denis Sheynikhovich, Ricardo Chavarriaga, Thomas Strösslin, Angelo Arleo, and Wulfram Gerstner, Laboratory of Computational Neuroscience, Brain Mind Institute, École Polytechnique Fédérale de Lausanne (EPFL), Lausanne, Switzerland.

Denis Sheynikhovich and Angelo Arleo are now at the Laboratory of Neurobiology of Adaptive Processes, Centre National de la Recherche Scientifique, Université Pierre \& Marie Curie, Paris, France. Ricardo Chavarriaga is now at the Institute of Bioengineering, École Polytechnique Fédérale de Lausanne (EPFL), Lausanne, Switzerland.

Correspondence concerning this article should be addressed to Wulfram Gerstner, Laboratory of Computational Neuroscience, Brain Mind Institute, École Polytechnique Fédérale de Lausanne (EPFL), Station 15, CH-1015, Lausanne, Switzerland. E-mail: wulfram.gerstner@epfl.ch
Further evidence has suggested that external sensory cues are used differently depending on the current strategy. The map-based, or locale, strategies seem to favor distal (e.g., landmarks attached to a maze walls) over proximal (e.g., intramaze objects) cues (Biegler \& Morris, 1993; Cressant, Muller, \& Poucet, 1997; Poucet, Lenck-Santini, \& Save, 2003). Moreover, configurations of distal cues are preferred over individual landmarks (Poucet et al., 2003; Suzuki, Augerinos, \& Black, 1980). In contrast, the S-R, or taxon, strategies preferentially use proximal cues, when they are available, as beacons that signal the goal location (Biegler \& Morris, 1993). In the absence of proximal cues, they fall back to distal-cue configurations (Eichenbaum, Stewart, \& Morris, 1990).

A particularly striking evidence for the control of behavior by configural cues has been observed during reorientation experiments in rectangular arenas (Cheng, 1986; Hermer \& Spelke, 1996; Margules \& Gallistel, 1988). In a typical experiment, a food-deprived animal is shown the location of a food source in a rectangular arena with distinct landmarks in the corners (Cheng, 1986). The animal is subsequently disoriented and is allowed to relocate the food source. Under these conditions, the animals exhibit systematic rotational errors, that is, they often go to the location that is diagonally opposite to the correct location. Since the correct and the diagonally opposite locations are indistinguishable with respect to the rectangular shape of the arena, these data suggest that the geometric layout of the arena, but not the identities of the corner landmarks, has been used by the animals during goal search. Preference for the geometric cues in this and similar 
experiments gave rise to the idea of a geometric module (Cheng, 1986; Gallistel, 1990), which is considered by many cognitive psychologists as a separate subsystem of the (vertebrate) animal brain, responsible for reorientation in a familiar environment (Cheng \& Newcombe, 2005; Wang \& Spelke, 2002).

In addition to the behavioral and lesion data, neurophysiological experiments have provided support for the separation of navigational strategies and for the importance of configural distal cues for navigation. Activity of place cells in the hippocampus of the rat is highly correlated with the location of the rat in the environment (Muller, Kubie, \& Ranck, 1987; O’Keefe \& Dostrovsky, 1971). The rat's position can be decoded with good accuracy from the activity of a hundred simultaneously recorded place cells (Wilson $\&$ McNaughton, 1993), suggesting that these cells store a representation of the environment, in agreement with the cognitive map concept underlying locale strategies. Although the precise mechanism by which place cells acquire spatial selectivity is not known, their tight anatomical and functional relations with the upstream population of grid cells provide an insight into how the spatially selective network may be organized (McNaughton, Battaglia, Jensen, Moser, \& Moser, 2006; O'Keefe \& Burgess, 2005; Solstad, Moser, \& Einevoll, 2006). Grid cells have been discovered in the dorsomedial entorhinal cortex (dMEC) one synapse upstream from the hippocampal area CA1, a principal area containing place cells (Fyhn, Molden, Witter, Moser, \& Moser, 2004). Grid cells are also spatially selective, but their firing fields are organized in a periodic triangular structure (a grid) covering the whole recording space (Hafting, Fyhn, Molden, Moser, \& Moser, 2005). Different cells have different spatial frequencies of their firing grids, so that a simple summation of their outputs by the downstream population can lead to a single-peaked activity akin to the Gaussian-like activity profile of a place cell (Samsonovich \& McNaughton, 1997). A one-synapse feed-forward network from grid cells to place cells is suitable to perform such a summation operation (O'Keefe \& Burgess, 2005; Solstad et al., 2006). This hypothesis is directly supported by the evidence that place cells in CA1 exhibit location-sensitive activity even without input from other areas, such as CA3 (Brun et al., 2002). Due to its remarkable layered organization and periodicity of firing fields, the grid-cell network has been functionally related to path integration (Fiete, Burak, \& Brookings, 2008; Hafting et al., 2005; O'Keefe \& Burgess, 2005), that is, the ability of an animal to integrate self-motion input (such as speed and direction of movement) over time (Etienne \& Jeffery, 2004). Since any efficient mapping system has to combine internal (self-motion) with external (e.g., visual) information during the process of map learning, the combined network of grid cells and place cells may be considered as an implementation of such a mapping system (McNaughton et al., 2006; O'Keefe \& Burgess, 2005).

In agreement with the data suggesting the importance of distal cues for locale navigation, place cells have been shown to rely on distal but not proximal cues. Rotation of a single polarizing cue card attached to the wall of a maze or of a set of objects located near the wall of the maze is followed by the corresponding rotation of place fields (Cressant, Muller, \& Poucet, 1999; Muller \& Kubie, 1987). In contrast, rotation of the same objects when located near the center of the maze fails to exert such a control (Cressant et al., 1997). Significantly, grid cells have also been shown to rotate their firing fields following the rotation of a distal cue (Hafting et al.,
2005). The importance of configural cues for place-cell firing follows from the experiments where place cells were recorded while the geometric layout of the environment changed (Gothard, Skaggs, \& McNaughton, 1996; O'Keefe \& Burgess, 1996). In these conditions, place cells either double (during environment stretching) or lose (during environment shrinking) their place fields. Moreover, grid cells rescale their firing fields in response to similar manipulations (Barry, Hayman, Burgess, \& Jeffery, 2007).

A challenge to the understanding of mechanisms of spatial navigation is to explain the behavioral data using available knowledge on anatomy and neurophysiology of neuronal networks mediating spatial memory and goal learning. Here, we address this challenge by proposing a computational neural model of navigation that provides a unifying point of view on the behavioral data described above and links these data to underlying neuronal properties. The model implements locale and taxon goal-navigation strategies and focuses on the influence of configurations of distal cues, represented by visual snapshots of the environment. The representation of the environment employed by the locale strategy is stored in a network of modeled grid and place cells, which link self-motion information with visual input. Such a combination allows for a direct comparison between the properties of modeled and real cells during environment manipulations, as well as between the model and animal behavior in navigational tasks where the location of a hidden target can be learned by different strategies. Moreover, reorientation behavior in rectangular arenas can also be analyzed using the same model, suggesting a set of experimental predictions concerning cell activity during reorientation behavior.

One of the central properties of the proposed model, namely, the use of visual snapshots as a principal source of external input, is closely related to the issue of the view-based matching approach to model navigation (Collett \& Collett, 2002). Such an approach has a long history in the study of insect navigation (see, e.g., Cartwright \& Collett, 1982, 1983) but recently attracted attention in relation to the study of human (Epstein, Graham, \& Downing, 2003; Gaunet, Vidal, Kemeny, \& Berthoz, 2001) and rat (Cheung, Stürzl, Zeil, \& Cheng, 2008; Stürzl, Cheung, Cheng, \& Zeil, 2008) navigation as well. In addition to the standard approach in which a similarity between snapshots is used directly to drive (taxon) behavior (Cheung et al., 2008; Collett \& Collett, 2002), our model suggests that snapshots can also be used to build an allocentric representation of the environment. As we show below, the properties of spatial representation built in this way may directly explain a number of neural (e.g., deformation of place fields in manipulated environments) and behavioral (e.g., rotational errors during reorientation) phenomena that were previously explained by assuming the existence of an additional process, such as, for example, the process of extracting distances to walls (Barry et al., 2006) or the process of extracting the environmental shape information from sensory input (Cheng, 1986).

\section{The Model}

In our model, the simulated rat moves through a virtual arena surrounded by walls. The size of the arena and visual features on the walls are chosen depending on the experimental paradigm. At each time step, the visual input is given by a snapshot of the environment processed by a large set of orientation-sensitive vi- 
sual filters, while the self-motion input is represented by the speed vector corresponding to the last movement. The motor actions are generated in the model by two separate pathways (see Figure 1). The first, taxon navigation pathway, associates visual input directly with motor actions and represents anatomical connections between the cortex and the dorsal striatum of the basal ganglia (caudate-putamen $[\mathrm{CP}]$ in the rat). The second, locale navigation pathway, generates actions based on a representation of space learned in a simplified model of place cells in the CA1 area of the hippocampus. The activity of model place cells encodes the location of the simulated animal and is further associated with motor actions, presumably encoded by the nucleus accumbens (NA) of the ventral striatum. The place cells receive feed-forward input from a population of simulated grid cells, similar to CA1 cells that receive direct input from grid cells in Layer II of the dMEC (Brun et al., 2002; Fyhn et al., 2004). The modeled grid cells perform integration of self-motion cues over time (i.e., path integration) and are influenced by visual input (Hafting et al., 2005). Further details of the model implementation are given below and are fully elaborated in the Appendix.

\section{Visual Input}

Visual snapshots are generated by a simple computer graphics algorithm (ray casting; Foley, van Dam, Feiner, \& Hughes, 1995) depending on the position and orientation of the simulated rat in the virtual environment (all environments used in our simulations are shown in Figure 2). Experimental evidence suggests that in the rat, (a) the variation in ganglion cell density is relatively small across the retina and the receptive field size of the cells is approximately constant (Kolb \& Tees, 1990) and (b) the vast majority of

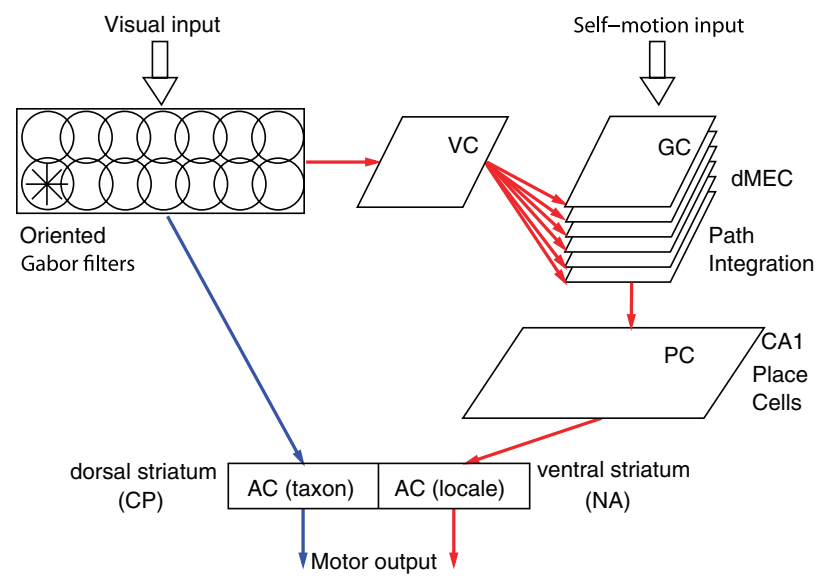

Figure 1. Model overview. Visual input is processed by a set of orientation-sensitive Gabor filters that project to the caudate-putamen (CP) of the dorsal striatum (blue arrows, taxon navigation pathway) and also to the hypothetical view cells (red arrows, locale navigation pathway). Grid cells (GC) in the dorsomedial entorhinal cortex (dMEC) receive selfmotion input and visual input, preprocessed by the population of view cells (VC). The GC connect to place cells (PC) in the hippocampal area CA1. The PC project to the nucleus accumbens (NA) of the ventral striatum. The dorsal and ventral parts of the striatum are modeled by two populations of action cells (AC). Cells in the $\mathrm{CP}$ encode a taxon strategy, while those in the NA encode a locale strategy. cells $(\sim 90 \%)$ in the primary visual cortex are orientation sensitive and the size of the center of their receptive field is $3^{\circ}-13^{\circ}$ in diameter (Girman, Sauvé, \& Lund, 1999). As a simplification, we model the output of the primary visual processing system as responses of a set of overlapping orientation-sensitive complex Gabor filters of width $\sigma_{g}=1.8^{\circ}$ (spatial wavelength $2 \sigma_{g}$ ) distributed uniformly across the view field $\left(300^{\circ}\right)$ using a rectangular sampling grid of $96 \times 12$ locations and eight orientations at each location. Examples of (simplified) filter representations of two snapshots from Virtual Environments $\mathrm{N}-\mathrm{I}$ and $\mathrm{B}-\mathrm{II}$ are shown in Figure 3B.

Snapshots (or their filter representations) represent spatial information in the egocentric (i.e., viewpoint-dependent) frame of reference. As such, they can be used to generate egocentric goaloriented actions, such as approaching a visible target. A simple example of such behavior would be to move forward while keeping the landmark with shape i (see Figure 3A, Environment B-II) in the center of the view field (assuming that the reward location is in front of that landmark). Note that in this case, no knowledge about the current head direction or position in the room is required to reach the goal. In our model, navigation in an egocentric reference frame is mediated by the taxon pathway (see below).

Visual snapshots in the model are also used to support behavior organized in an allocentric reference frame (i.e., fixed with respect to the environment), mediated by the locale navigation pathway. During exploration of a novel environment, snapshots of unfamiliar views are stored in hypothesized view cells. The activity of a view cell $i$ at time $t$ depends on the similarity between the momentary pattern of filter activities at time $t$ and the pattern stored in cell $i$. The activity is maximal if the current view matches the stored view. Note that views taken from the same location but with different directions of gaze (i.e., different head directions) look very different. Therefore, to measure the similarity between views, we apply the following three-step procedure: First, we estimate the momentary head direction based on information currently available in the population of view cells (see Appendix, Equations A10 and A11); second, we rotate the representation of the current view by the difference in head direction between the current and the stored views; third, we evaluate the difference between the filter activities of the rotated and the stored views (see Appendix, Equation A12). Under the assumption that the current head direction has been estimated correctly, view cells code for the location in the environment in allocentric coordinates (see Figure 3C). Nevertheless, the egocentric aspect of views shows up in the fact that the activity of the view cells is maximal if the current gaze direction coincides with the one used during initial exploration. View cells in our model represent memory of local views that seem to be stored (in humans) outside of the hippocampus (Gillner \& Mallot, 1998; Spiers, Burgess, Hartley, Vargha-Khadem, \& O'Keefe, 2001).

\section{Goal-Oriented Behavior}

Motor actions are encoded in the model by two hypothesized populations of action cells that represent motor-related output of $\mathrm{CP}$ or $\mathrm{NA}$, respectively. The $\mathrm{CP}$, in particular its lateral part (Devan \& White, 1999), is thought to be involved in the development of S-R behavior, in which a set of stimuli is repeatedly associated with a rewarded motor response (Packard \& McGaugh, 


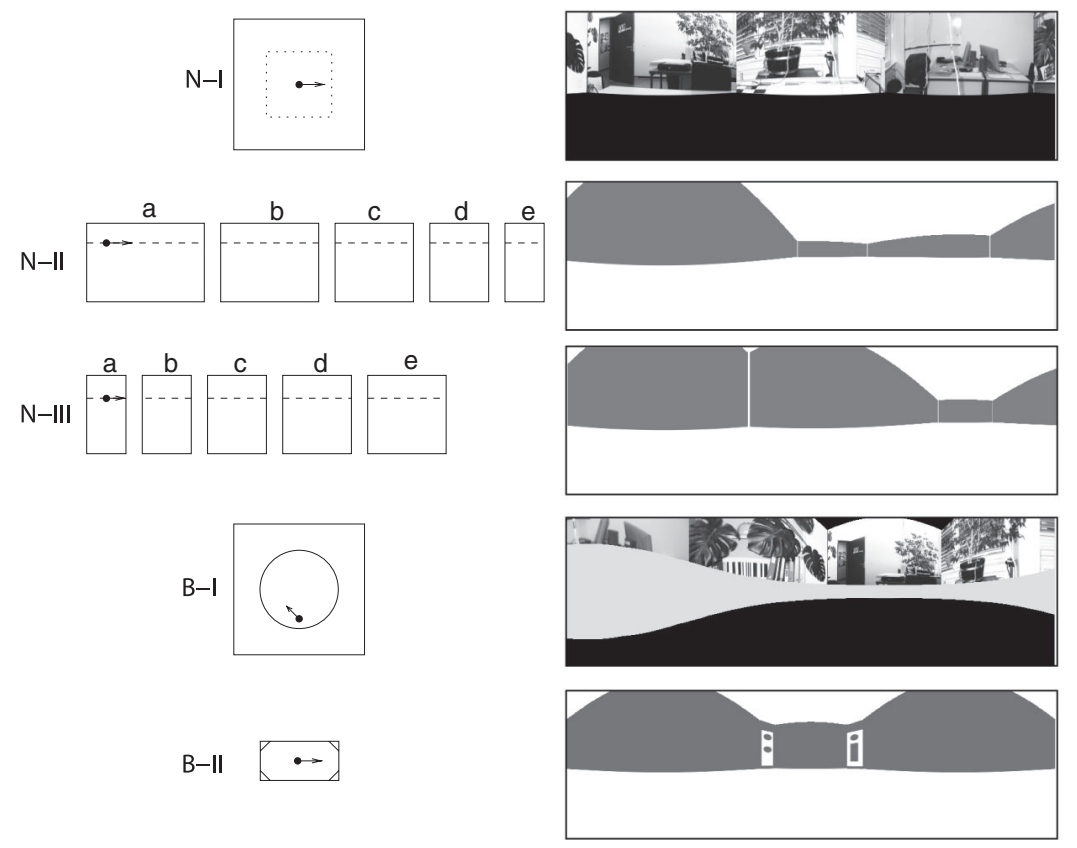

Figure 2. Testing environments used in computer simulations. In each row, the right part of the figure shows an example snapshot of the room, and the left part of the figure shows the top view of the virtual room. The black dot and the arrow show the position and direction at which the snapshot was taken. The dotted line in the top view of Room N-I marks the area accessible to the model rat in this environment. The dashed line in Rooms $\mathrm{N}-$ IIa through $\mathrm{N}-\mathrm{II}$ and $\mathrm{N}-\mathrm{III}$ a through $\mathrm{N}-\mathrm{IIIle}$ marks a linear trajectory of the model rat. The circle in the top view of Room B-I marks the border of the simulated water maze. Environment B-II is used in the simulations of the experiment of Cheng (1986).

1992, 1996; White \& McDonald, 2002). The NA of the ventral striatum receives direct projection from the CA1 area of the hippocampus (Kelley \& Domesick, 1982) and has been hypothesized to associate location information with reward and emotional information to produce goal-directed motor behavior (Brown \& Sharp, 1995; Redish, 1999). Lesions of the CP and NA differentially impair the animal's ability to learn S-R or spatial tasks, respectively (Annett, McGregor, \& Robbins, 1989; Packard \& McGaugh, 1992; Sutherland \& Rodriguez, 1990).

In agreement with these data, action cells in our model of $\mathrm{CP}$ receive direct feed-forward input from the visual filters, while action cells in the modeled NA receive input from place cells (see Figure 1). In each population, there are many action cells, and the activity of each action cell encodes a different direction of movement. The preferred directions of the action cells in a population span $360^{\circ}$, such that the population activity can be treated as a distributed code for the movement direction. An important difference between the two action-cell populations is that the activity of the action cells in $\mathrm{CP}$ is considered to encode the egocentric rotation angle, while the activity of the cells in NA is treated as a code for the allocentric direction of movement.

In a typical scenario involving the taxon strategy, the simulated rat is placed in an environment and receives visual input in the form of a set of visual filter activities corresponding to the currently perceived snapshot. The visual input results in an activation of action cells in the $\mathrm{CP}$, which is interpreted by the simulated rat as a motor command to turn by the resulting angle. As a simplification, we do not model the movement of the rat along the resulting direction but simply assume that the rat would move straight until it hits an obstacle. If the chosen direction is correct, the rat would reach the goal (e.g., the hidden platform in a water maze) and receive a reward, otherwise it will hit a wall (no reward).

In a typical locale strategy scenario, the simulated rat is placed in an environment, and an estimation of the current allocentric location and head direction is given by the activity of place cells (described below). The place-cell activity results in an activation of motor cells in the NA, interpreted by the rat as a motor command of moving in an allocentric direction encoded by the action-cell activity. After the movement is performed (the extent of the movement is defined as the constant speed of the rat multiplied by the size of the time step; see Appendix, Table A1), the new location will correspond to a different pattern of place-cell activity, resulting in the next movement, and so on until the goal is reached (or the trial time is over). Upon reaching the goal, a reward is given.

The trajectory of the rat in a particular trial and hence the success or failure in reaching the goal in that trial are fully determined by the synaptic strengths (connection weight values in the model) between the visual filters and action cells in the $\mathrm{CP}$ (taxon strategy) and between the place cells and action cells in the NA (locale strategy). Learning consists in adjusting the weight values to maximize performance. The theoretical framework of reinforcement learning (Sutton \& Barto, 1998) suggests how the weight values may be learned by an online reward-based algorithm that minimizes the difference between the predicted and received 

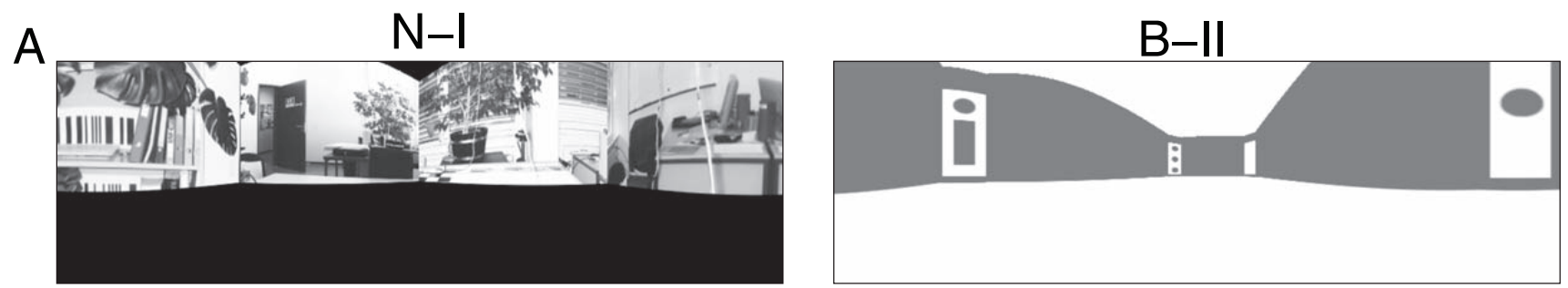

$\mathrm{B}$
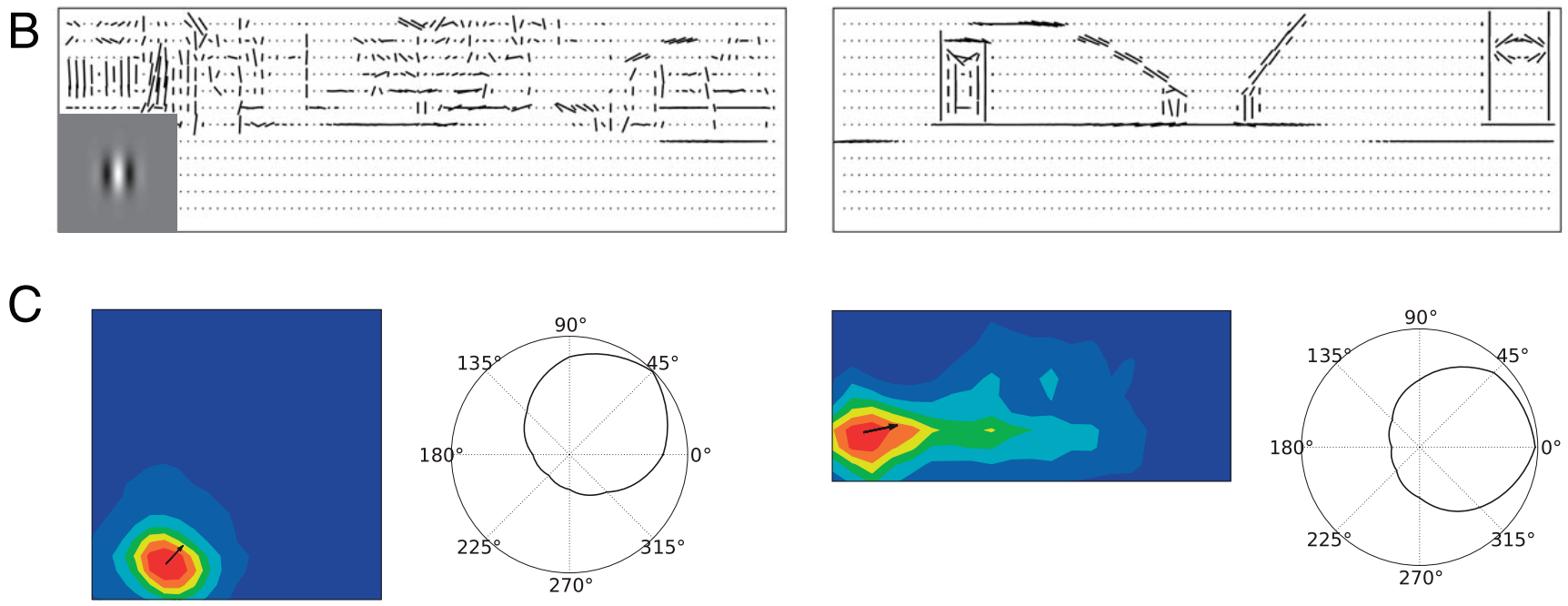

Figure 3. Processing of visual input in Simulated Environments N-I (left column) and B-II (right column). A: Example snapshots with $300^{\circ}$ horizontal view field. B: Responses of visual filters applied to the input snapshots shown in A. Filter responses are drawn as black lines centered at different positions in the filter grid. The length of a line is proportional to the mean amplitude of eight Gabor filters with different orientations centered at the point (zero amplitude is shown as a black dot). The orientation of the line is determined by the mean orientation of the filters, weighed by the corresponding amplitudes. Mean values are shown for clarity; all filter amplitudes are used in the model without averaging. Inset shows an example of a Gabor filter sensitive to a vertical edge in its receptive field (not to scale) C: Firing maps show the receptive fields of view cells that store snapshots shown in A, calculated with exact allocentric head direction and averaged over all orientations. Arrows show the position and orientation of the simulated rat at which the corresponding snapshots were taken. Polar plots show firing rate of the view cells as a function of head direction. The activity is maximal for $\Phi=47^{\circ}(\mathrm{N}-\mathrm{I})$ or $\Phi=$ $11^{\circ}$ (B-II), which corresponds to the head direction of the stored view.

rewards (see Appendix). Such a reward prediction error is thought to be encoded in the activity of the dopaminergic neurons in the substantia nigra pars compacta $(\mathrm{SNc})$ and ventral tegmental area (VTA) of the basal ganglia (Schultz, 1998; Schultz, Dayan, \& Montague, 1997). Experimental evidence has suggested that dopaminergic neurons in the $\mathrm{SNc}$ project to the $\mathrm{CP}$, that lesions of SNc specifically impair S-R behavior (Da Cunha et al., 2003, 2006), and, moreover, that dopamine input seems to be required for plasticity to take place in the cortico-striatal synapses (Pawlak $\&$ Kerr, 2008). The NA receives dopaminergic input from the VTA (Sesack \& Pickel, 1990), and lesions of NA produce deficits in the hidden, but not visible, version of the water-maze task (in naive rats; Annett et al., 1989). Here, we do not model explicitly SNc and VTA but simply assume that the reward prediction error signal (in the form of the $\delta$ variable in Equation A4 in the Appendix) arrives at the synapses between the visual filters and $\mathrm{CP}$ action cells (or between the place cells and NA action cells) after an action has been performed.

\section{Spatial Representation}

The evidence for the involvement of the hippocampus in navigation (Morris, Garrud, Rawlins, \& O'Keefe, 1982; O'Keefe \& Nadel, 1978) is complemented by behavioral data suggesting its role in latent learning (O'Keefe \& Nadel, 1978), path integration (Whishaw \& Maaswinkel, 1997), and development of stimulusstimulus associations (White \& McDonald, 2002). On the neural level, these data have been related to the properties of place cells in the areas CA3-CA1 of the hippocampus, since their firing fields develop during unrewarded exploration (Hill, 1978; Wilson \& McNaughton, 1993), persist in darkness (Quirk, Muller, \& Kubie, 1990), and depend on multiple sensory stimuli (Gothard et al., 1996; O’Keefe \& Burgess, 1996).

However, dMEC grid cells, directly upstream from the CA1, have been shown to possess similar properties. Namely, firing fields of the grid cells were shown to develop rapidly during exploration, persist in darkness, and rotate their firing fields fol- 
lowing rotation of visual cues (Fyhn et al., 2004; Hafting et al., 2005). Moreover, direct feed-forward projections from the dMEC to CA1, bypassing dentate gyrus and CA3, are sufficient to produce place fields (Brun et al., 2002). These data suggest that place cells in CA1 may inherit their spatially correlated firing from the grid cells (O'Keefe \& Burgess, 2005; Solstad et al., 2006).

In our model, several grid-cell populations encode the position of the simulated rat in the environment, while place cells represent simple feed-forward readout of the grid-cell activity (see Figure 1). More specifically, recurrent connectivity in each grid-cell population results in localized activity packets that change their positions according to internally generated speed and direction information. The hardwired pattern of connectivity is chosen such that firing fields of the cells in each population are periodic, and the translation of the speed information into the movement of activity packets is chosen to produce triangular grids of experimentally observed spacing, orientation, and field size (see Figures 4D and 4E). Place cells are recruited during exploration by rapid Hebbian learning, that is, a new cell is connected to all strongly active grid cells and the connection weights are initialized by the presynaptic activities. Simple summation of the activities of presynaptic grid cells with different spatial frequencies of their grids results in a Gaussian-like receptive field of a single place cell (see Figure 4B; McNaughton et al., 2006; O'Keefe \& Burgess, 2005; Solstad et al., 2006). This is consistent with the evidence showing that hippocampal neurons perform linear summation of their synaptic inputs (Cash \& Yuste, 1999; Gasparini \& Magee, 2006).

Grid cells in the model are influenced by visual input, which is preprocessed and stored in the view cells mentioned above. In visually nonambiguous environments, spatially close locations

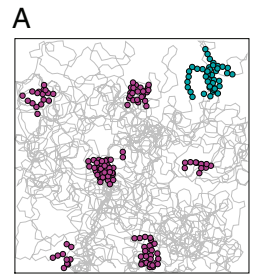

D

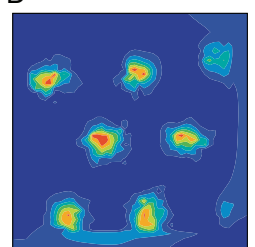

B

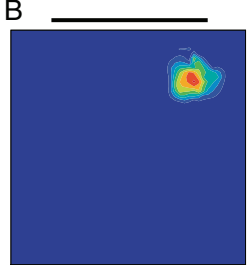

E

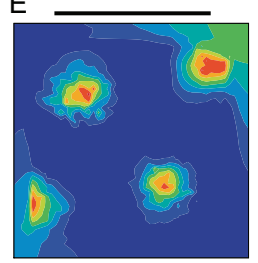

C

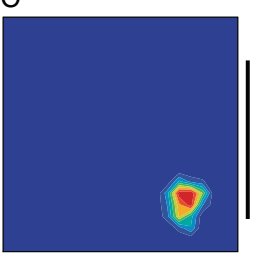

F

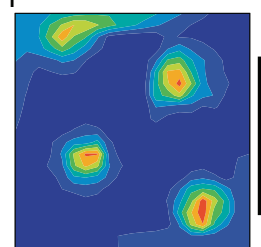

Figure 4. Firing fields of modeled grid cells and place cells. A: Trajectory of the modeled rat during testing phase in a square simulated environment with superimposed locations where the firing rates of one modeled place cell (green) and one modeled grid cell (violet) were higher than 0.7 of their maxima over the whole environment. B, C: Color-coded rate maps of the place cell shown in A during testing in the normal condition (B) and when all visual cues were rotated $90^{\circ}$ clockwise $(C$; the black bar denotes cues on the northern wall in the nonrotated environment). D, E: Rate maps of two cells from grid-cell populations with indices $n=2$ (D; grid orientation $3^{\circ}$, the same cell as shown in A) and $n=4(\mathrm{E}$; grid orientation $9^{\circ}$ ) during testing. F: Rate map of the cell shown in $\mathrm{E}$ with all cues rotated $90^{\circ}$ clockwise. share similar visual features, and therefore, a subset of simultaneously active view cells represents a particular location in the environment. This allocentric position code is used in the model to correct a mismatch between the path integration and visual input resulting from a cumulative error in the path integrator or from changes in visual environment. The correction mechanism is implemented in the form of connections between the view cells and grid cells. The connection weight between a grid cell and a view cell is set according to the Hebbian rule, that is, it is high if the cells are simultaneously active above a certain threshold and low otherwise. Once the weights are set, an activity profile in the view-cell population will induce an allocentric location signal in each of the grid-cell populations, resulting in a shift of the grid-cell activity packets toward the visual estimate (see Appendix, Equations A13 and A14, for details).

Visual input plays an important role upon the entry to a familiar environment. More precisely, when the simulated rat is placed in a familiar environment, the positions of the activity packets in the grid-cell populations are set according to the visual input. Such an initialization of the path-integration network by the visual input represents a recall of the familiar environment by the simulated rat (Samsonovich \& McNaughton, 1997).

\section{Results}

The following three sections describe the model behavior in different experimental paradigms. The first set of simulations described properties of model neurons along the locale navigation pathway, focusing on the changes in their activity following changes in the geometric layout of the experimental arena. The second set of simulations addressed the ability of the model to perform goal search in water-maze-like simulated environments. The last set of simulations investigated how the geometric layout of the environment can influence goal-oriented behavior and related our results to the concept of the geometric module introduced in the beginning of the article. The results of the three sets of simulations taken together suggest that a model without a geometric module is able to account for the influence of the environment shape on grid cells, place cells, and behavior.

\section{Simulation 1: Deformation of Place Fields and Rescaling of Grid-Cell Firing Patterns}

The hypothesis of place-field formation by direct summation of presynaptic activity of grid cells is attractive because of its simplicity (McNaughton et al., 2006; O'Keefe \& Burgess, 2005; Solstad et al., 2006). However, it is not clear whether this hypothesis can explain the dependence of the place-field shape on the geometry of the environment (Gothard et al., 1996; O'Keefe \& Burgess, 1996). It is equally unclear how the rescaling of the grid-cell firing patterns in response to environment changes (Barry et al., 2007) can be put in agreement with the deformation of hippocampal place fields.

Place-field formation by summation of grid-cell activities predicts a comodulation of place and grid cells in response to environmental changes. Animals can sense these changes via visual input, which can in turn influence place cells and grid cells. Since place cells and grid cells are driven not only by vision but also by path integration, the interaction between these two types of sensory 
input is likely to play a role in the induction of changes in the cell activity in response to environmental changes (Byrne, Becker, \& Burgess, 2007; Samsonovich \& McNaughton, 1997). Hence, we first illustrate the interaction between visual input and path integration in our model in a fixed familiar environment and then turn to the analysis of the activity of place and grid cells in stretched and shrunk environments.

Method. In this simulation, five different computer experiments were conducted. In Experiment 1, the activities of place and grid cells were analyzed, while the simulated rat was moving in quasi-random directions within a square experimental arena of $1 \mathrm{~m}$ $\times 1 \mathrm{~m}$ located in the middle of a large room with multiple visual features (see Environment N-I, Figures 2 and 3). Before the analysis of place fields was performed, exploration was simulated by allowing the simulated rat to visit uniformly distributed locations and orientations in the environment for 3,000 time steps, which correspond to about $6 \mathrm{~min}$ of exploration. At each time step, a newly recruited view cell memorized a corresponding snapshot of the environment. Similarly, a newly created place cell memorized the active subset of grid-cell population (see Appendix for details). After all 3,000 locations were visited, the weights between view cells and grid cells were set according to the cross-correlation rule (see Appendix, Equation A13), capturing the essence of Hebbian learning: Cells that are active together become connected with stronger weights (Kali \& Dayan, 2000; Samsonovich \& McNaughton, 1997). To test whether the model could reproduce the rotation of the firing fields in response to the rotation of visual cues (Hafting et al., 2005; Muller \& Kubie, 1987), the cue rotation was simulated by interrupting the simulation, rotating all the visual cues by $90^{\circ}$ clockwise, and restarting the simulation from a different location.

In Experiments 2-5, with shrinking and stretching environments, two series of rectangular rooms were used (N-II and N-III; see Figure 2). In each series, the first room (N-IIa and N-IIIa) is referred to as the original environment and the other rooms as shrunk $(\mathrm{N}-\mathrm{II})$ or stretched $(\mathrm{N}-\mathrm{III})$ versions of the original room. All rooms had the same width $(0.86 \mathrm{~m})$, and their lengths were $1.72 \mathrm{~m}, 1.42 \mathrm{~m}, 1.12 \mathrm{~m}, 0.82 \mathrm{~m}$, and $0.52 \mathrm{~m}$ for the N-II series and $0.52 \mathrm{~m}, 0.66 \mathrm{~m}, 0.82 \mathrm{~m}, 0.96 \mathrm{~m}$, and $1.12 \mathrm{~m}$ for the N-III series. The lengths were chosen to approximate the real experimental conditions (Gothard et al., 1996; O'Keefe \& Burgess, 1996). Each room had gray walls $0.6 \mathrm{~m}$ high and a white floor and ceiling (see Figure 2).

In Experiment 2, a set of shrinking rectangular environments (N-II) was used, and the simulated rat was running back and forth along a line parallel to the northern wall of the enclosure, simulating movement along a linear track. During the exploration phase, the simulated rat explored the linear track in the original environment (N-IIa) similar to the exploration phase of Experiment 1 , except that the head direction was either $0^{\circ}$ or $180^{\circ}$ according to the direction of movement. In the testing phase, the simulated rat was exposed to each of the shrinking environments (N-IIb through $\mathrm{N}-\mathrm{II}$ ) in turn; place-cell activities were analyzed while the simulated rat was moving along the track in these novel environments. Exploration and testing phases in Experiment 3 were identical to those in Experiment 2 except that this time, the series N-III of stretching environments was used.

In Experiment 4, the series N-II of shrinking environments was used; the exploration phase was similar to that in Experiment 2, but now the simulated rat was allowed to move in two dimensions. During the testing phase, it moved in a zigzag fashion through the testing boxes such that directional dependence of the twodimensional place fields could be assessed. Exploration and testing phases in Experiment 5 were identical to those in Experiment 4 except that this time the series N-III of stretching environments was used.

In the model, learning of a new environment assumes that grid-cell firing patterns are fixed with respect to the environment during an initial exploration (the assumption is used to calculate connection weight values between view cells and grid cells; see Appendix, Equation A13). This assumption is supported by the recording data of Hafting et al. (2005) showing that entorhinal grid cells exhibit stable firing patterns from the outset of exploration in complete darkness for as long as $20 \mathrm{~min}$. Such a remarkable stability of firing suggests that even in the absence of visual input, firing grids of entorhinal cells are fixed to the environment, possibly by using other sources of external input (Maaswinkel \& Whishaw, 1999; Save, Nerad, \& Poucet, 2000) in combination with a particular exploration strategy (Whishaw, Hines, \& Wallace, 2001). It was shown previously that suitable exploration strategies involving return to previously visited places lead to a stable learning of the connections from view to place cells even in the presence of a noisy path integrator (Arleo \& Gerstner, 2000). Since for the purposes of the present article, we were not interested in exploration strategies per se, we simply fixed the firing grid to the environment by setting noise in the path integrator to zero during exploration. During testing, we simulated the cumulative error by setting the error in the path integrator to $10 \%$ of the displacement and rotation from the previous step.

To analyze changes in population activity of place cells induced by the environment deformation, we compared the population firing in deformed environments to that in the original environment in Experiments 2 and 3. A coefficient of cross-correlation between the population firing patterns (or population vectors) in the deformed and original environments served as a measure of similarity (Gothard et al., 1996). If $r_{i}^{\mathrm{pc}}(x)$ denotes the firing rate of place cell $i$ at location $x$ in a deformed environment and $r_{i}^{\mathrm{pc}}(y)$ denotes the firing rate of the same cell at location $y$ in the original environment, the coefficient of cross-correlation between the population vectors is given by

$$
c_{x y}=\frac{\sum_{i} r_{i}^{\mathrm{p} c}(x) r_{i}^{\mathrm{p} c}(y)}{\sqrt{\sum_{k}\left(r_{k}^{\mathrm{pc}}(x)\right)^{2}} \sqrt{\sum_{l}\left(r_{l}^{\mathrm{p} c}(y)\right)^{2}}},
$$

where the denominator ensures that the coefficient of crosscorrelation of two identical population vectors $c_{x x}=1$. Each element of the cross-correlation matrix $\left\|c_{x y}\right\|$ shows how similar population vectors are in a deformed and the original environments at locations $x$ and $y$, respectively.

Results and discussion. Our simulations showed that grid cells and place cells in the model exhibit firing patterns similar to those recorded in real neurons. After learning, these firing patterns are stable in time despite the fact that the modeled path integration is subject to cumulative errors. This stability is due to the associative connections between visual snapshots (stored in the view cells) and grid cells learned during exploration. These associative connections can also explain the deformation of place fields and 
rescaling of grid-cell firing patterns in stretching or shrinking rectangular environments.

During the testing phase of Experiment 1 (square room), the cumulative error in the path integrator was corrected by visual input (represented by visual snapshots of the environment) both in the population of grid cells (see Figures 4A, 4D, and 4E) and the population of place cells (see Figures 4A and 4B). Rotation of visual cues was followed by the rotation of the firing pattern of grid cells (see Figure 4F) and place cells (see Figure 4C), in agreement with experimental data (Hafting et al., 2005; Muller \& Kubie, 1987). In the model, the rotation of place fields is a direct consequence of the initialization of the position of the grid-cell activity packet according to the visual input from view cells.

In shrinking and stretching environments (Experiments 2-5), place cells that had fields near walls in the original environment kept their fields near the walls in the novel environments for both one-dimensional and two-dimensional movement regimes (see, e.g., Cells 1 and 4, Figure 5A, and Cell 1, Figure 5B). In contrast, cells with peak firing near the middle lost their fields in the shrunk environments (see Cells 2 and 3, Environments N-IId and N-IIe, Figure $5 \mathrm{~A}$ ), whereas their fields became doubly peaked in the stretched environment (see Cell 2, Environment N-IIIe, Figure
5B), similar to biological CA1 cells (Gothard et al., 1996; O'Keefe \& Burgess, 1996; Redish, Rosenzweig, Bohanick, McNaughton, \& Barnes, 2000).

To check that the same effects could be observed on the level of the whole place-cell population, we compared the population activity in the deformed environments with that in the original environment (for one-dimensional movement, Experiments 2 and 3 ). The similarity of the population firing, expressed as the crosscorrelation between the firing rates at each position in the original environment and those at each position in the deformed environment (see Method, above), is illustrated in Figure 6. The disappearance of place fields in shrunk environments corresponds on the cross-correlation plots to the zero correlation between the population firing rates near the middle of the original environment (see Figure 6A, N-IId and N-IIe). The doubling of the place fields in strongly stretched environments can be seen by observing that when the simulated rat moves from left to right (see Figure 6B, $\mathrm{N}-\mathrm{III}$ and $\mathrm{N}-\mathrm{III}$, top), the high-correlation band crosses the middle (dotted) line earlier than the rat reaches the middle of the stretched environment; the same happens when the simulated rat moves in the opposite direction (see Figure 6B, N-IIId and N-IIIe, bottom). Therefore, the resulting place field, averaged over the two

A

Shrinking

cell 1 cell 2

cell 3

cell 4

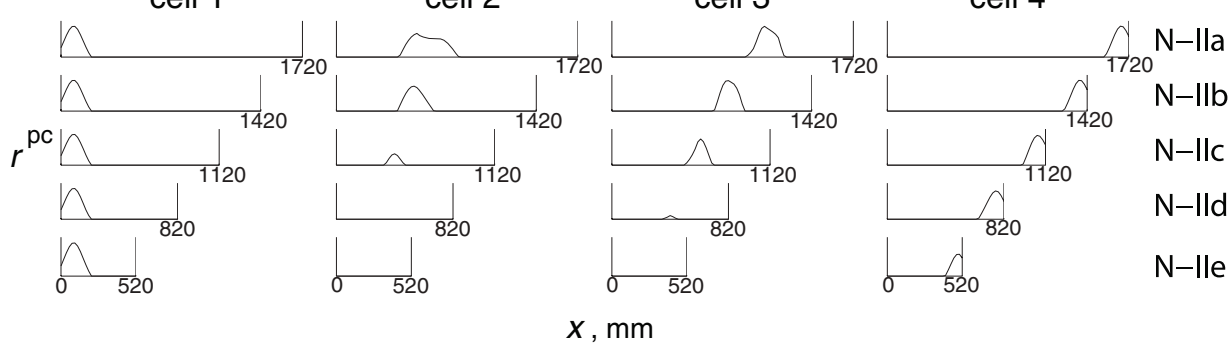

B

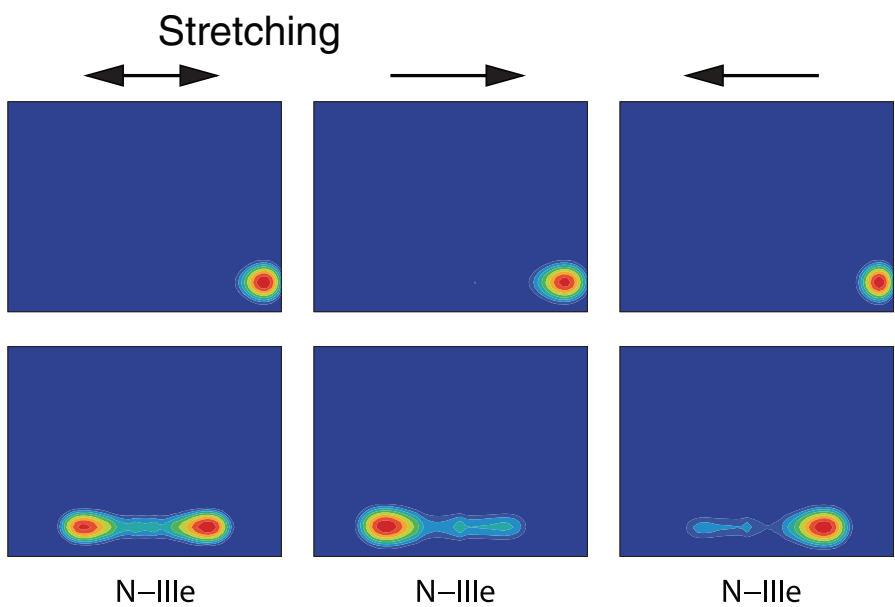

Figure 5. Place fields in shrinking and stretching environments. A: Place fields (firing rate as a function of the simulated rat position on the track) of four modeled CA1 cells in the original (N-IIa; top row) and shrunk (from top to bottom: N-IIb, N-IIc, N-IId, N-IIe) environments during the rightward movement. B: Two-dimensional place fields of two different place cells for the original (N-IIIa) and stretched (N-IIIe) versions of the box for leftward movement (right), rightward movement (second right), and averaged across the two directions (second left). The direction of movement is shown by the black arrow on top of the plots. 
A
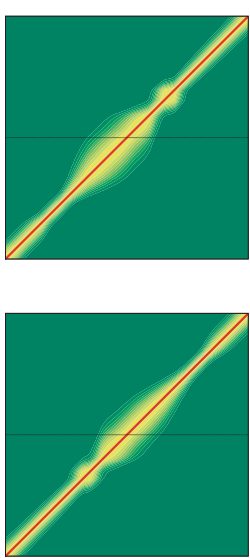

$\mathrm{N}-1 \mathrm{la}$
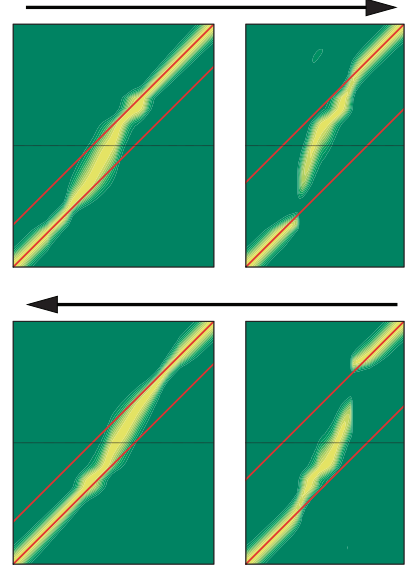

$\mathrm{N}-\mathrm{Ilb}$

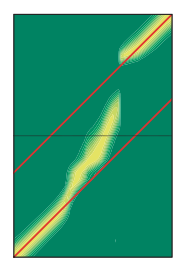

$\mathrm{N}-\mathrm{IlC}$
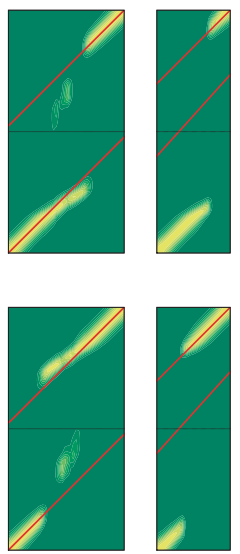

N-lld

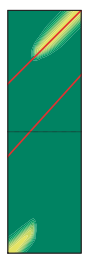

$\mathrm{N}$-Ile

B
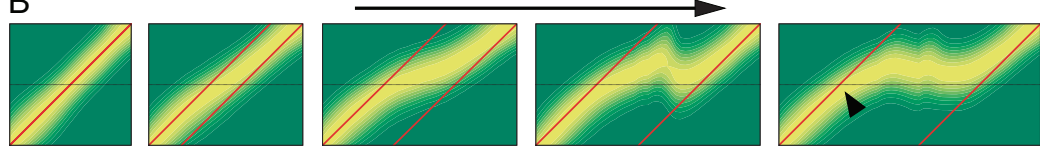

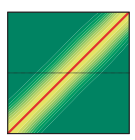

$\mathrm{N}-$ IIIa

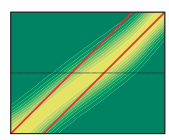

$\mathrm{N}-\mathrm{IIIb}$

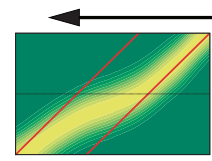

$\mathrm{N}-$ IIIC

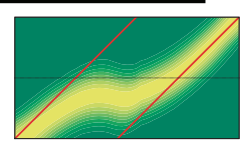

N-IIId

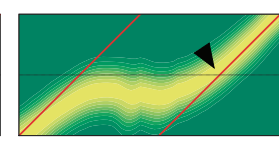

N-llle

correlation

1.0

Figure 6. Similarity of population firing patterns in the original and deformed environments. Each plot shows cross-correlation $c_{x y}$ between firing rate vectors of the place-cell population (yellow $=$ high correlation, green $=$ low correlation; see Method section of Simulation 1) that correspond to position $y$ of the simulated animal in the original environment (vertical axis) and its position $x$ in the deformed environment (horizontal axis). The red lines correspond to the locations of the cross-correlation maxima if the population firing were determined only by path integration. The black dotted line corresponds to the middle of the original environment. In all plots, a cell that, in the original environment, has its place field near the middle, in the deformed environment will have its place field shifted to the position corresponding to the crossing between the middle line and the highcorrelation band. A: Cross-correlation matrices for the original (N-IIa) and four shrunk (N-IIb through N-IIe) environments for two directions of movement (shown by the black arrow on top of the plots). Cells near the middle of the original environment lose their place fields when the deformation is strong, as shown by the zero correlation of firing rates near the middle line for Environments N-IId and N-IIe. B: Cross-correlation matrices for the original (N-IIIa) and four stretched ( $\mathrm{N}-\mathrm{IIIb}$ through $\mathrm{N}-\mathrm{III}$ ) environments. Cells near the middle of the original environment double their place fields when the deformation is strong (Environments N-IIId and N-IIIe). During rat movement from left to right, cells in the middle fire closer to the left wall in the stretched track (small arrow in the top panel, Environment N-IIIe), whereas they fire closer to the right wall during movement from right to left (small arrow in the bottom panel, Environment N-IIIe).

directions of movement, consists of two components, the activation of which will depend on the movement direction of the simulated rat (Samsonovich \& McNaughton, 1997).

Stability of place fields near walls is explained in our model by similarity of visual (snapshot) information in the original and deformed environments when the simulated rat is close to the walls. In this case, the visual input is strong enough to control the position of the place fields. However, when the rat is moving at a long distance from the walls, both path integration and visual input contribute to place-cell firing. Path integration in the model works by shifting the activity packet in a grid-cell layer from one group of cells to the next. In the case of deformed environments, visual input either speeds up (in shrunk environments) or slows down (in stretched environments) the movement of the activity packets. Speeding up of the activity packets results in narrow place fields, while slowing them down widens the fields. Very strong deformation results in disappearance or doubling of place fields (Samsonovich \& McNaughton, 1997). Strong shrinking causes the activity packets to lose their coherence with downstream place cells due to periodicity in their firing locations, making the place fields disappear. Strong stretching of the environment causes asymmetry of stretched place fields: When moving from left to 
right, the left part of the field is more active, whereas, during the leftward movement, the right part of the field is more active (O'Keefe \& Burgess, 1996).

Next, we looked at the grid-cell activity during two-dimensional movement in the deformed environments (Experiments 4 and 5). For a small amount of shrinking or stretching, we observed rescaling of the firing grids (see Environments N-IIb and N-IIIb, Figure 7) in agreement with the data from Barry et al. (2007). However, a

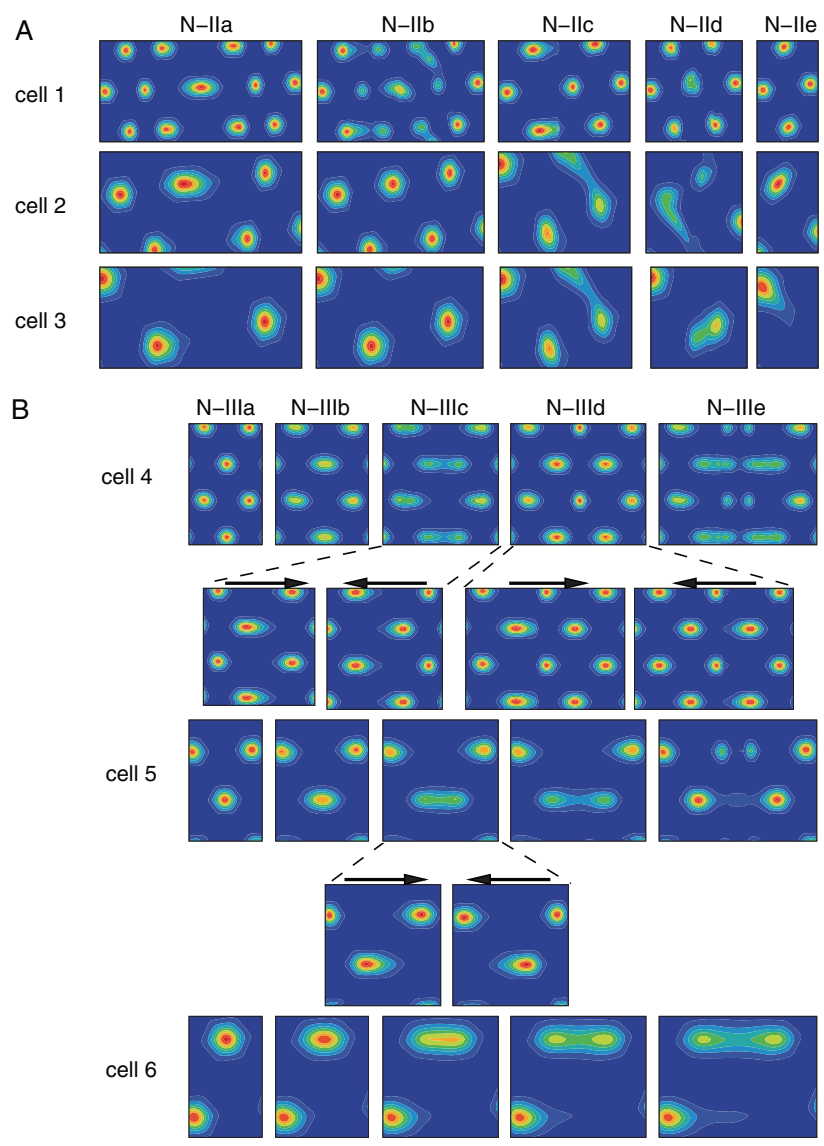

Figure 7. Rescaling of the firing pattern of a modeled grid cell in response to environment deformation. A: Firing fields of three grid cells from populations $n=2,4,6$ (from top to bottom, the grid orientations are $3^{\circ}, 9^{\circ}$, and $15^{\circ}$, respectively) in the training environment (N-IIa) and four shrunk environments ( $\mathrm{N}-\mathrm{IIb}$ through $\mathrm{N}-\mathrm{II}$; amount of shrinking relative to the training environment $0.83,0.65,0.48$, and 0.30 , respectively). B: Firing fields of three different grid cells from populations $n=1,2,4$ (from top to bottom, the grid orientations are $0^{\circ}, 3^{\circ}$, and $9^{\circ}$, respectively) in the training environment (N-IIIa) and four stretched environments (N-IIIb through $\mathrm{N}-\mathrm{IIIl}$; amount of stretching relative to the training environment is $1.27,1.57,1.81$, and 2.15 , respectively). Insets for Cells 4 and 5: firing fields of the same cells for different movement directions. Rescaling is observed for the smaller amount of deformation (shrinking: N-IIb; stretching: N-IIIb). Stronger deformation results in disappearance of firing fields near the middle of the environment (shrinking: N-IIc through $\mathrm{N}-\mathrm{IIe}$ ) or doubling of the fields near the middle (stretching: N-IIId and N-IIIe). For an intermediate amount of stretching, the double fields exhibit a dependence on the direction of movement (Cells 4 and 5, N-IIIc); however, the directional dependence disappears for even stronger stretching (Cell 4, N-IIId). stronger deformation resulted in disappearance (shrinking: $\mathrm{N}-\mathrm{IIC}$ through N-IIe, Figure 7A) or doubling (stretching: N-IIId through N-IIIe, Figure 7B) of firing fields near the middle of the environment. Moreover, for some amounts of stretching, the double fields became asymmetric, similar to the firing fields of downstream place cells (see Figure 7B, insets for Cells 4 and 5, Environment $\mathrm{N}-$ IIIc). In contrast to the place fields, the asymmetry in the grid-cell firing disappeared for even stronger deformation (see Figure 7B, inset for Cell 5, Environment N-IIId). The latter effect is due to the periodicity of the firing fields: When the spatial frequency of the double fields becomes equal to the spatial frequency of the grid, the double field loses its directional dependence. The disappearance and doubling of grid-cell firing fields were not reported by Barry et al. (2007), possibly due to the fact that they analyzed only one shrunk (amount of shrinking was 0.70 relative to the baseline) and one stretched (amount of stretching was 1.43 relative to baseline) environment. In our model, the disappearance and doubling effects on the level of grid cells were observed for stronger shrinking (i.e., for environments shrunk to less than $65 \%$ of the size of the original environment) and stronger stretching (i.e., stretched to more than $157 \%$ of the original size; see Figure 7).

Thus, experimental data on place-field deformation in shrunk or stretched environments can be explained by visual feature processing, from visual input to grid cells and from grid cells to place cells, without recognition of walls or explicit calculation of distances to walls. In this case, the apparent influence of the geometric layout on place- and grid-cell activity (which is sometimes taken as an evidence for the importance of the shape of surrounding space for spatial processing in relation to the problem of the geometric module; see Wang \& Spelke, 2003) is accounted for by the mismatch correction between visual and self-motion cues. Such a mismatch correction mechanism per se is independent from any geometry-related information (as in, e.g., Experiment 1 of the present set of simulations) but is expressed in Experiments 2-5 in a way that might be interpreted as influence of geometry. On the level of grid cells, our model predicts that (a) grid-cell rescaling should be observed even in the absence of functional connections between grid cells and place cells and (b) switching visual input off during a recording session in a deformed environment should eliminate the rescaling effect.

We note here that our explanation of the place-field shape deformation and grid rescaling depends heavily on the presence of external sensory cues that make the information about layout changes available to the rat. Therefore, in the case where external sensory cues are removed, the model predicts that path integration will take control over place fields for a longer time compared to the case when external cues are present. This explanation is consistent with the data of Gothard, Hoffman, Battaglia, and McNaughton (2001) showing that in a shrinking linear track, place fields are aligned with the (movable) starting position longer in the dark than in the light.

\section{Simulation 2: Hidden Goal Navigation in the Water Maze}

In this set of simulations, we examined learning of goal-oriented strategies in the model. For this purpose, we simulated two watermaze tasks in which rats had to learn the location of a hidden goal. The Morris water-maze task with variable starting locations (see 
Figure 8A) has been shown to depend critically on the hippocampus (Morris et al., 1982) and is generally considered a standard test of spatial memory (McDonald, Hong, \& Devan, 2004). Animals trained in this task can immediately generalize to novel starting locations (Morris, 1981), suggesting that they have acquired a representation of spatial layout of the surrounding environment. However, when started from the constant starting location (see Figure 8B) in each trial, animals with hippocampal lesions were almost as successful as normal rats (Eichenbaum et al., 1990), suggesting that a different memory system was used in this case, which might store the S-R association between the extramaze cues visible from the start and the heading toward the hidden platform (Da Cunha et al., 2006). Further experiments have shown the dependence of such an S-R behavior on brain areas along the taxon pathway, that is, CP (Packard \& McGaugh, 1992, 1996; White \& McDonald, 2002) and SNc (Da Cunha et al., 2003, 2006). In contrast to the hippocampus-dependent locale strategy, taxon strategies produce stereotyped trajectories, as demonstrated by the inability of fornix-lesioned rats to find the hidden platform from a novel starting position (Eichenbaum et al., 1990).

Method. Both the variable-start and constant-start tasks were simulated in a square room of $2 \mathrm{~m} \times 2 \mathrm{~m}$ with high walls and multiple visual cues. The experimental arena, located in the center of the room, was surrounded by a gray circular wall $1.2 \mathrm{~m}$ in diameter and $0.2 \mathrm{~m}$ high so as to simulate the wall of the water maze (see Environment B-I, Figure 2). An invisible target area 6 $\mathrm{cm}$ in diameter located in the southwest quadrant of the simulated water maze served as a hidden goal.

Before training (i.e., during the exploration phase), the simulated rat explored the water maze without the platform to learn place fields, similar to the previous simulation. After exploration, the simulated rat could use either taxon or locale navigational strategy to learn the goal location. Ten different rats were simulated, and results were averaged across the 10 simulated animals.

In our model, learning of the two strategies occurs in the synapses between the visual filters and action cells in CP (for the taxon navigation) and between place cells in the hippocampus and action cells in NA (for the locale navigation). Similarly to the dorsal-striatum- and hippocampus-dependent pathways in the rat (Devan \& White, 1999; Packard \& McGaugh, 1996; White \& McDonald, 2002), the locale and taxon pathways in the model are independent, and therefore, they can learn in parallel and compete for control of behavior. A simplified model of competition was adopted in the simulations: In each trial, the simulated rat used two strategies to reach the hidden platform in two separate runs. A strategy was considered either successful (coded by 1) or not successful (coded by 0 ) depending on a performance criterion (see Figures 9A and 9C). The winning strategy for each trial was determined by a running average, based on the number of successes in the 10 preceding trials. Such a simple competition scheme allows for a separate analysis of intact versus lesioned simulated rats. An intact animal is assumed to always choose the winning strategy, while a lesioned animal can only use a strategy that is not affected by the lesion.

In Experiment 1, simulated rats were tested in the variable-start condition. During training, the simulated animals learned to go to the invisible platform. A training trial started by placing the simulated rat in one of the four starting positions (see Figure 8A), chosen at random in the beginning of the trial. The initial orientation of the simulated rat was randomly chosen between $0^{\circ}$ and $360^{\circ}$. If the goal was hit (during the locale strategy run) or the head direction after rotation was straight to the platform (for the taxon strategy run), the simulated rat received a positive reward $(R=1)$. Wall hits during locale strategy runs were negatively rewarded $(R=-0.5)$.

In Experiment 2, the rats were tested in the constant-start condition. The training was identical to that in Experiment 1, except that the same position $\mathrm{S}$ was used in each trial (see Figure 8B). After training was completed, the rats were tested from novel starting positions. All weights in the model were kept fixed, and the simulated rats were given 100 testing trials from each of the positions $\mathrm{W}$ and $\mathrm{E}$ not used during training.

Results and discussion. To explore goal-oriented behavior of the model, we simulated the Morris water-maze task with variablestart and constant-start conditions. In both conditions, the simulated rat was able to learn the task. We analyzed the role of the locale and taxon strategies for both conditions.

Simulation results showed that in the variable-start condition, the locale strategy was the winner across all training trials and quickly reached a high average success rate (see Figure 9D, left). In contrast, the success rate of the taxon strategy did not increase significantly during training, suggesting that the taxon strategy was
A Variable-start task

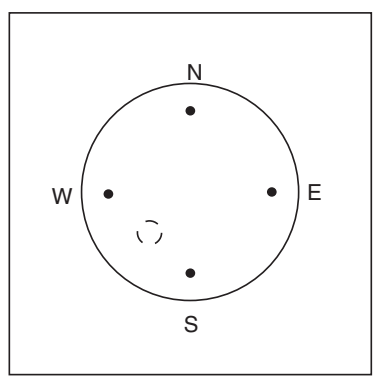

B Constant-start task

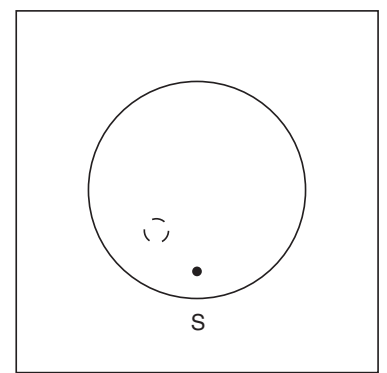

Figure 8. Experimental setup for two water-maze tasks. The large circle represents the top view of the circular water maze. The black dots mark the starting positions in the maze (denoted N, E, S, and W). The small dotted circle marks the area of the hidden platform in the simulated maze. A: Variable-start condition (Morris, 1981). B: Constant-start condition (Eichenbaum et al., 1990). 
not able to learn this task (see also Figure 9C, left). Thus, the model is consistent with the experimental data showing that intact animals rely strongly on the locale strategy in this task and that lesions of the hippocampus (or NA) disrupt learning, whereas lesions along the taxon pathway (i.e., $\mathrm{SNc}$ or $\mathrm{CP}$ ) do not produce any impairment (Da Cunha et al., 2006; Morris et al., 1982). Moreover, an analysis of the escape latency (see Figure 9A, left) revealed that when using locale strategy, the simulated rat learned rapidly during the first four to five trials and achieved, after approximately 15 trials, an asymptotic performance (Morris, 1981).

In the constant-start condition, both strategies were able to learn the location of the hidden platform (see Figure 9D, right). These results are consistent with the evidence that the task can be solved by intact animals as well as animals with a lesioned fornix (i.e., the axon bundle that connects hippocampus to NA; Eichenbaum et al., 1990). An analysis of the escape latency and heading distributions supported further these results (see Figures 9A, right, and 9C, right). In addition, learning of the taxon strategy was slower at the beginning of training, while its performance was superior to that of the locale strategy after prolonged training, suggesting that for an intact animal the preference for the taxon strategy increases with experience (Packard \& McGaugh, 1996).

To examine the ability to generalize to novel starting locations, the simulated rat was tested from two novel starting positions (W and E) after the training in the constant-start condition (S). When the simulated rat was using the locale strategy, it generalized immediately to the novel starting locations, as shown by the comparison of the average escape latencies from the novel and familiar locations (see Figures 10A and 10B). The reason for the good generalization ability in the case of the locale strategy is that during training the simulated rat had a possibility to learn the direction of swimming to the platform from most of the locations in the maze, despite the fact that it started always from the same location (as illustrated by the action map acquired by the simulated rat during the constant-start condition; see Figure 9B, right). In contrast, the taxon strategy was disrupted by novel visual cues, such that, in all trials, the direction to the platform was estimated incorrectly (see Figures 10A and 10C).

Together, these results demonstrate that configurations of distal cues, encoded in visual snapshots, can be successfully used to learn to approach a hidden platform from variable starting locations using a hippocampal position code (Morris, 1981), as well as to learn the direction to the platform in the constant-start condition using direct sensory-motor associations (Eichenbaum et al., 1990). The two simulated memory systems function independently and mediate the allocentric (locale) and egocentric (taxon) navigation strategies. In the variable-start condition, the form of spatial memory acquired during learning is flexible in generating novel paths, while, in the constant-start condition, the memory is limited to a fixed set of S-R associations and thus produces stereotyped behavior (O'Keefe \& Nadel, 1978).

\section{Simulation 3: Reorientation in Rectangular Environments}

According to the geometric module hypothesis (Cheng, 1986; Hermer \& Spelke, 1996; Wang \& Spelke, 2002), an animal reorients itself upon entry into a familiar environment using solely the geometric shape of the environment, but not other, nongeometric features, such as textures, colors, odors, or visual landmarks. Since the shape information must be somehow extracted from the sensory input, the conceptual brain module (Fodor, 1983) that extracts it must discard the nongeometric information present in the sensory input or, equivalently, be impenetrable to it (Gallistel, 1990).

This hypothesis is based on two main experimental results originally described by Cheng (1986) for rats and later reproduced with other species (see Cheng \& Newcombe, 2005, for review). The first one is derived from a working memory task and consists in the observation (Cheng, 1986) that rats often make rotational errors when they try to relocate a previously found food in a rectangular arena with distinct landmarks in the corners (see Figure 11A). More precisely, in this experiment, rats searched for food near the correct location in $46 \%$ of trials and near its diagonally opposite location in $28 \%$ of trials (they searched far from both locations during the remaining $26 \%$ of trials; see Figure 11C). The pattern of errors did not change even after extensive training. This result was interpreted as a preference for the room geometry information over the landmark information during reorientation. The second result is that in the same environment but in a different task (reference memory task; see Figure 11B), the rats could use landmarks to identify goal location. When rats were trained to go always to the same corner from the center of the box, the percentage of rotational errors decreased gradually to $\approx 22 \%$ compared to $\approx 76 \%$ of correct trials (Cheng, 1986). This and similar results have usually been interpreted as evidence that although the nongeometric cues can be used after specialized training, they are not used during reorientation, supporting the impenetrability argument (Cheng, 1986; Hermer \& Spelke, 1996; Wang \& Spelke, 2002).

Results of the simulations performed in the previous section suggested a different interpretation of these data. Since, in the first task of the experiment of Cheng (1986; see Figure 11A), different starting positions were used in each trial, the only successful strategy is the locale strategy. The taxon strategy cannot be applied in this case due to the lack of stable sensory-response associations linked with reward. Hence, the rotational errors, as well as their stability over time, might have been caused specifically by the application of the locale strategy. However, since the second task (see Figure 11B) permits a stable association of cues and reward, the rats could use a taxon strategy in this task, which might explain the observed decrease in the number of rotational errors. Why are rotational errors more prominent in the locale strategy than in the taxon strategy?

A crucial difference between the two strategies is that the locale strategy encodes actions in an allocentric frame of reference (i.e., a coordinate system that is fixed with respect to external cues), while the taxon strategy does so in an egocentric one (i.e., with respect to the current view). Since all actions performed by the organism are inherently egocentric, the locale strategy requires a coordinate transformation, and to perform this transformation, current allocentric position and head direction need to be estimated. In contrast, for taxon strategies, such a coordinate transformation is not needed, since all actions are performed with respect to the currently visible cues.

In our model, the allocentric position and head direction are determined during a reorientation phase by matching the currently perceived snapshot (represented by the responses of the orientation-sensitive visual filters to the input image) with snapshots stored in memory during exploration (see Appendix, Equa- 


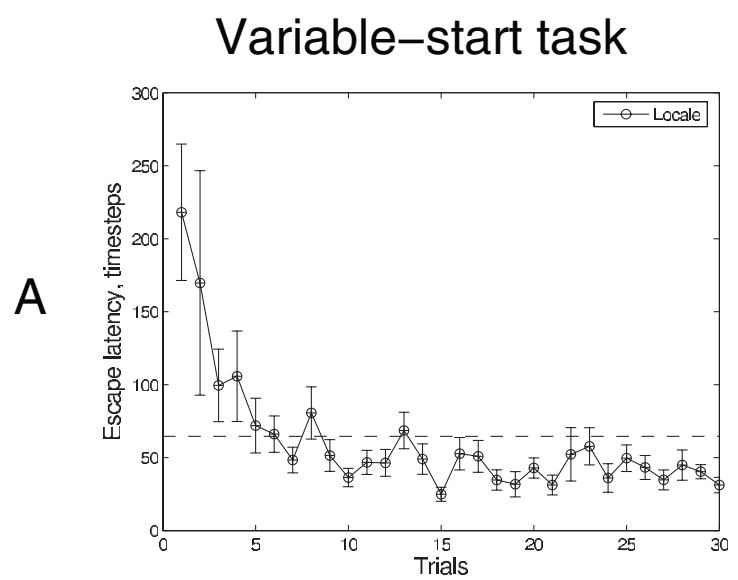

Constant-start task

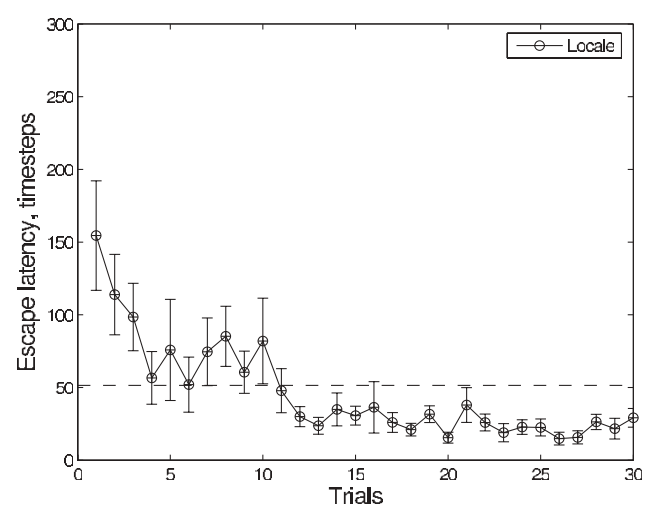

B
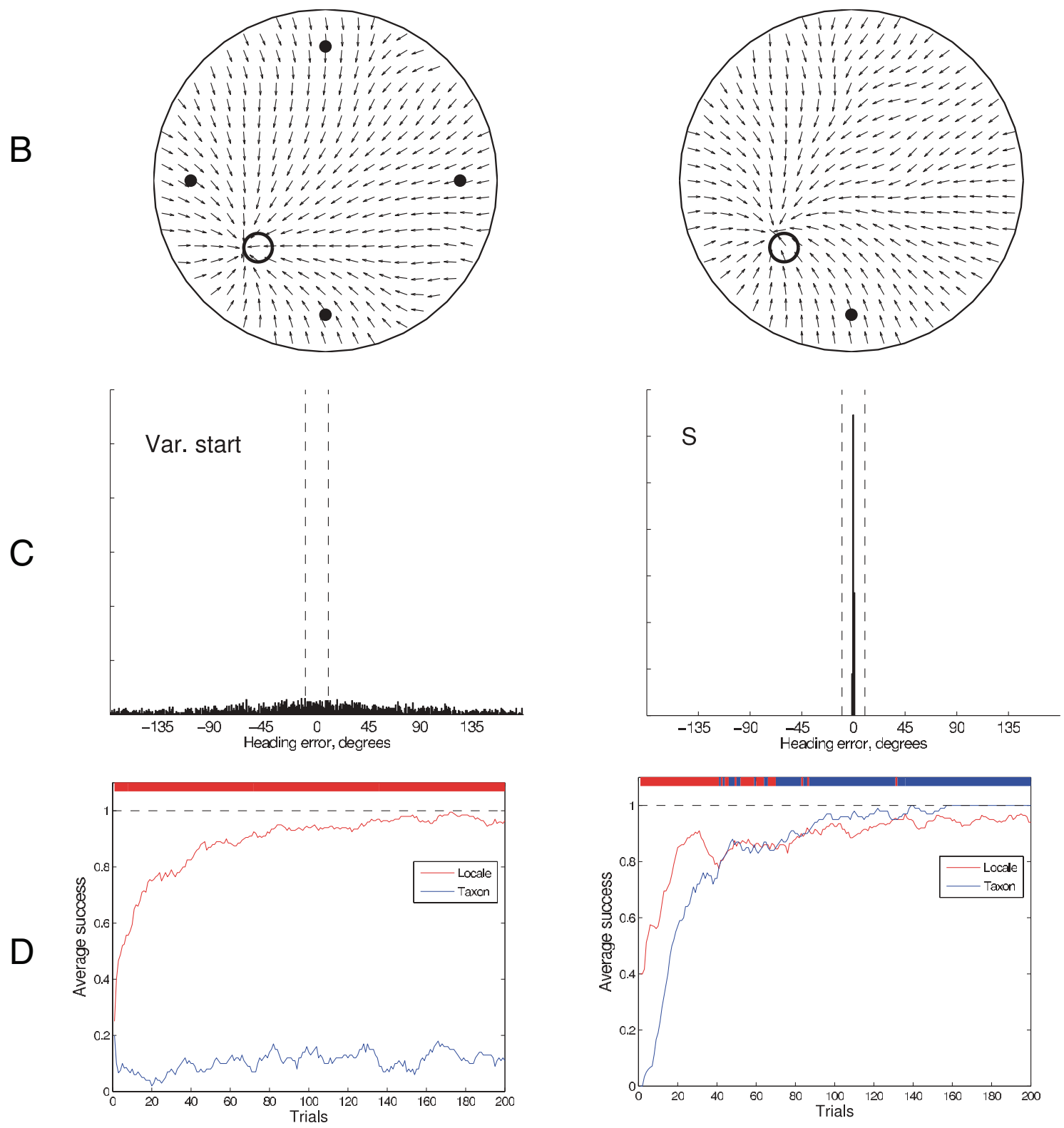

Figure 9 (opposite). 
tions A10-A14). In a rectangular environment with symmetrically arranged landmarks, as well as in the environments where one of the walls has a different color (Cheng, 1986; Hermer \& Spelke, 1996), the snapshots taken in opposite directions from rotationally opposite locations are highly similar (in terms of the difference between their filter representations). Hence, the rotational errors can be caused by the ambiguity of visual cues in the process of snapshot matching. The following set of simulations was designed to check whether the key experimental results from Cheng (1986) could be reproduced by our model, that is, without an explicit geometry-related processing module.

Method. The simulations were conducted in a virtual environment, designed in analogy to the setup used in the experiment of Cheng (1986), and consisted of a rectangular arena with gray walls and distinct landmarks in the four corners. The arena size was $1.2 \mathrm{~m} \times 0.6 \mathrm{~m}$ with walls $0.6 \mathrm{~m}$ high (see Environment B-II, Figures 2 and 3).

Similar to the water-maze experiment described above, 10 animals were simulated, and two experimental conditions were used: variable start and constant start. The two conditions corresponded to the working memory and reference memory experiments of Cheng (1986), respectively.

Experiment 1 aimed to show that the reorientation procedure, required by the locale strategy in the variable-start condition, is subject to rotational errors caused by the ambiguity in the visual input during snapshot matching. After the standard exploration phase (i.e., for 3,000 time steps), 1,000 reorientation trials were performed. In each trial, the simulated rat was placed at one of five starting positions as denoted in Figure 11A. Initial orientation was randomly chosen between $0^{\circ}$ and $360^{\circ}$. Once placed at the starting location, the simulated rat performed a reorientation procedure, that is, the current allocentric heading was estimated from the set of local views stored during the exploration phase and the path-integration network was reset accordingly (see Appendix, Equations A10-A14). The outcome of a reorientation trial was considered as correct when the absolute value of the difference between the estimated and real allocentric headings was less than $20^{\circ}$, as a rotational error when the difference was greater then $160^{\circ}$, and as a miss otherwise. The same procedure as above was repeated in the cue-rich Environment $\mathrm{N}-\mathrm{I}$ and the symmetric Environment N-IIIa (with quasi-random starting positions) to estimate the dependence of the number of rotational errors from the number of polarizing visual cues.

To measure spatial receptive fields of view cells (for one simulated animal), we placed the simulated animal at a set of locations distributed over a regular grid in each of the simulated environments (B-II, N-I, and N-IIIa). At each point of the grid, the simulated animal was oriented at eight different orientations. For each position and orientation, the reorientation procedure was performed as described above, and the activities of all view cells were calculated with the estimated allocentric heading.

Experiment 2 was designed analogous to the reference memory task of Cheng (1986) with a fixed starting condition. Our simulations tested the suggestion that switching to taxon strategy can decrease the number of rotational errors when the simulated rat starts always from the same position in the box. In this experiment, the simulated rats were trained to turn toward the same landmark from the same starting position (the center of the box) across all training trials. The initial orientation of the simulated rat was randomly chosen between $0^{\circ}$ and $360^{\circ}$ at the start of the trial. This phase was performed identical to the taxon training in Simulation 2. The reward was given when the heading of the simulated rat after the end of the trial was not more than $\pm 10^{\circ}$ off from the direction toward the landmark in the northwest corner (i.e., the simulated rat performed a correct turn). Otherwise, no reward was given. If the resulting heading was within $\pm 10^{\circ}$ of the direction to the rotationally opposite corner, it was considered a rotational error. The ratio between the number of correct turns and that of rotational errors was calculated for each trial, averaged over animals, and smoothed with 100-trial-kernel.

Results and discussion. We simulated the working memory experiment of Cheng (1986) that led to the hypothesis of the geometric module. The results of simulations showed that reorientation errors in the model correspond well to the rotational errors observed by Cheng (see Figure 11C). We found that reorientation was correct in $45 \%$ of trials and resulted in a rotational error in $27 \%$ of trials (in the remaining $28 \%$ of trials, the estimated heading was neither correct nor rotationally opposite).

We next asked the question how our model would perform in environments that have either more or fewer polarizing cues than the environment used in the experiment of Cheng (1986). We found that the simulated rats made no orientation errors in the cue-rich Environment N-I, since snapshots taken in different directions can be distinguished well by the visual system (see Figure 11C). In contrast, the heading was estimated correctly as often as a rotational error was made in a perfectly symmetric rectangular Environment N-IIIa.

The crucial role of the view-based heading estimation for the simulation results is illustrated in Figure 12. Estimation of heading in the simulated environment of Cheng (1986) and in the symmetric environment is subject to rotational errors, as seen from the doubly peaked histograms (see Figure 12A, B-II and N-IIIa). The rotational errors in Environments B-II and $\mathrm{N}-\mathrm{III}$ a are caused by

Figure 9. Simulation results of the variable-start (left column) and constant-start (right column) water-maze tasks. A: Evolution of the escape latency for the locale strategy. Trials where the escape latency was below a threshold (dashed line) were considered successful for the locale strategy. The threshold was chosen as $\mu_{\text {stab }}+\sigma_{\text {stab }}$, where $\mu_{\text {stab }}$ and $\sigma_{\text {stab }}$ are the average values of the escape latency and its standard deviation when the performance stabilized (in the present simulations, after 20 trials). B: Action maps acquired by the locale strategy during training. The arrows show learned directions to the platform (open circle) from the sample locations. The black dots mark starting positions of the simulated rat. C: Distributions of heading errors during 200 training trials for the taxon strategy. Zero error corresponds to the case when the simulated rat heads directly toward the center of the platform. Trials where the direction estimation error was within $10^{\circ}$ (dashed lines) were considered successful for the taxon strategy. D: The curves show, for each trial, the average number of successes across 10 preceding trials for the locale (red) and taxon (blue) strategies. The colored bar at the top of the plot shows the winning strategy. In the variable-start condition, the locale strategy is winning (left). In the constant-start condition, both strategies have similar success rates, but eventually, the taxon strategy takes over (right). 
A
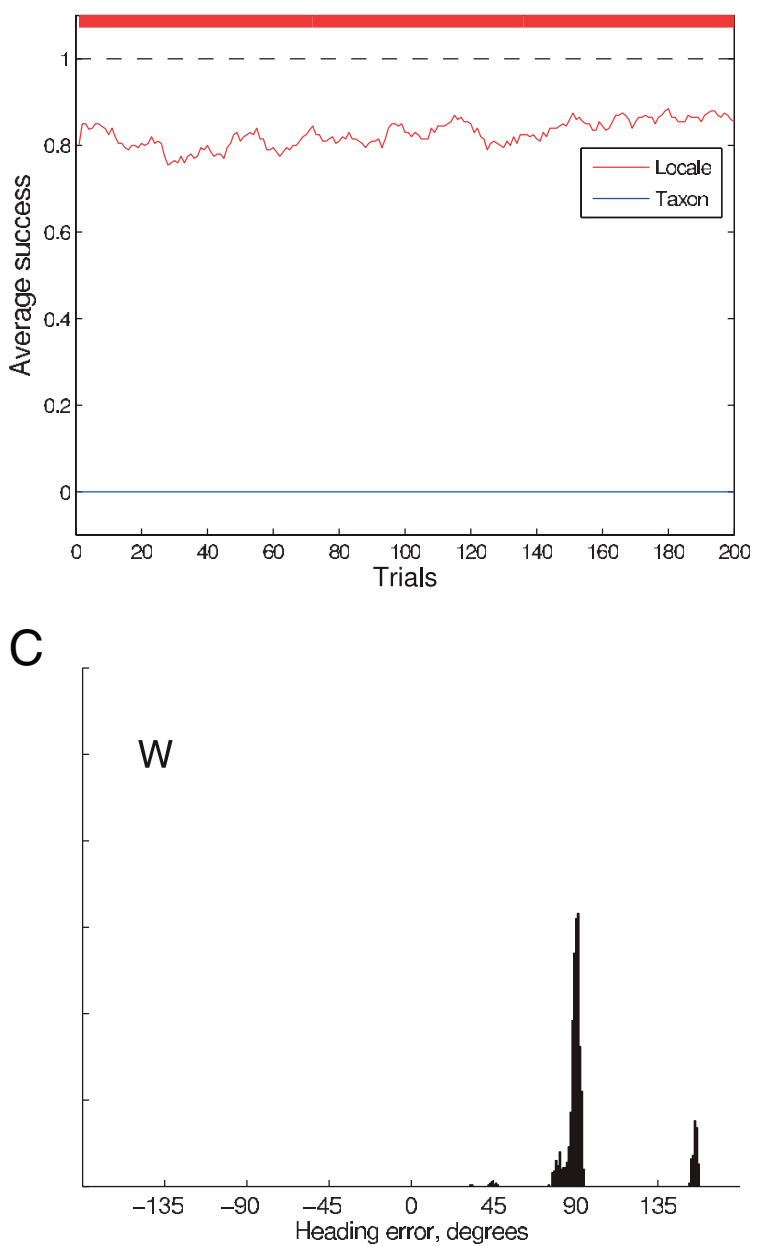

B
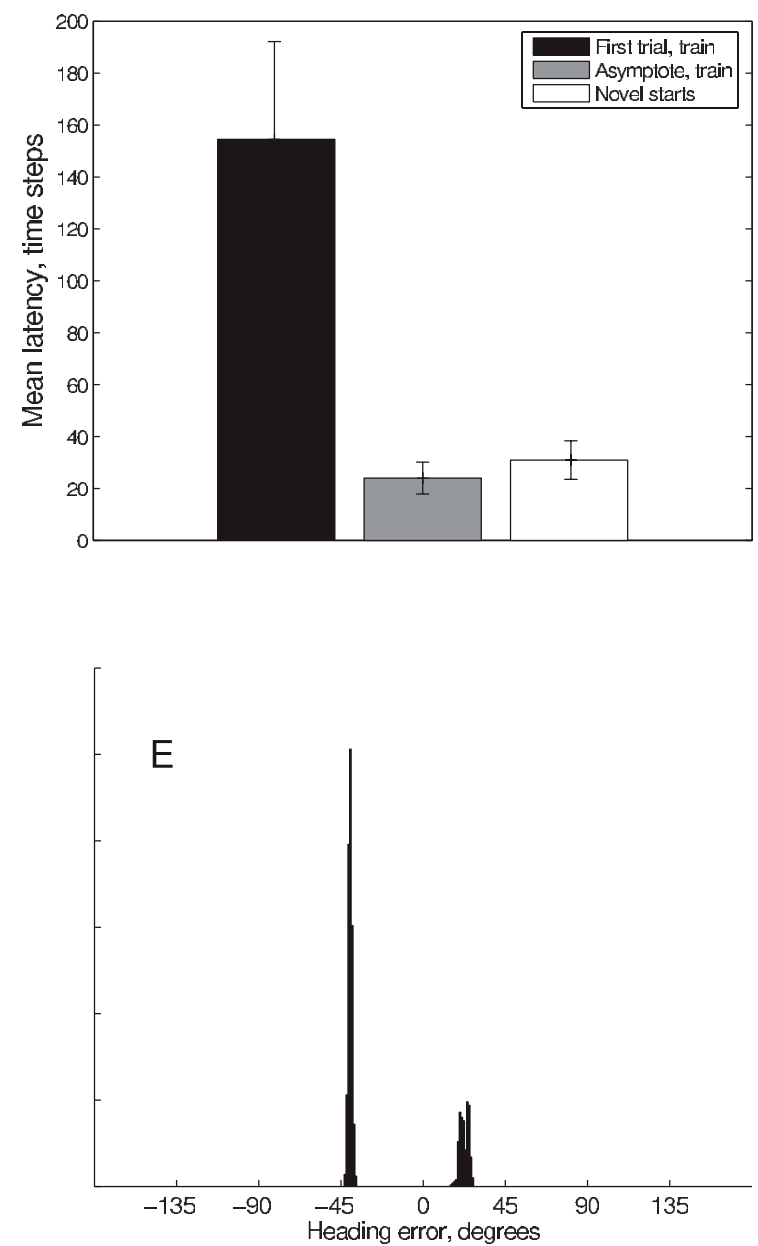

Figure 10. Novel-start tests. A: Success rates for the locale (red) and taxon (blue) strategies in 200 testing trials from starting positions $\mathrm{W}$ and $\mathrm{E}$. The taxon strategy fails, leaving the locale strategy as a clear winner. B: Mean escape latency $\pm S E$ from the novel starting positions for the locale strategy (white) in comparison to the latency of the first training trial (random search; black) and the asymptotic latency (gray) for the variable-start task. C: Distributions of estimated directions to the platform for the taxon strategy, measured during 200 trials from the novel starting positions $\mathrm{W}$ (left) and $\mathrm{E}$ (right). Zero is aligned with the direction to the platform. Bin size is $1^{\circ}$.

the similarity of visual snapshots taken from rotationally opposite locations and are hence due to the (nearly) symmetric layout of the environment. In the cue-rich environment, however, where such a symmetry does not exist, no rotational errors were observed (see Figure 12A, N-I).

Rotational errors made during heading estimation lead to rotational errors during self-localization. In the model, this is illustrated by the doubly peaked spatial receptive fields of view cells in Environment B-II and in the symmetric environment (see Figure 12B, B-II and N-IIIa, respectively). The part of the receptive field in the location rotationally opposite to the correct one (marked by the arrows in Figure 12B) is caused by the rotational errors in heading estimation. Note that the receptive fields are single peaked if the heading is always estimated correctly (see Figure 3C). We emphasize that the activity in the view-cell population is always single peaked and corresponds to either the correct or rotationally opposite position, depending on the heading error (see Figures $12 \mathrm{C}$ and $12 \mathrm{D}$ ). The population activity can be interpreted as the internal estimation of the current position by the animal and suggests that when the rotational error is committed, the animal thinks that it is at the position rotationally opposite to the correct one. On the basis of these results, we propose that the error in heading estimation ultimately causes the animal to search for a goal in a place diagonally opposite to the actual food location (Cheng, 1986; Margules \& Gallistel, 1988; Pearce, Good, Jones, \& McGregor, 2004).

Although all three environments considered in our simulations were rectangular, the percentage of rotational errors with respect to the total number of errors changed from 0 (Environment $\mathrm{N}-\mathrm{I}$ ) to $\approx 50 \%$ (Environment B-II) to $\approx 70 \%$ (Environment N-IIIa). Such a gradual increase in the number of orientation errors upon a reduction of the number of polarizing visual cues indeed suggests 

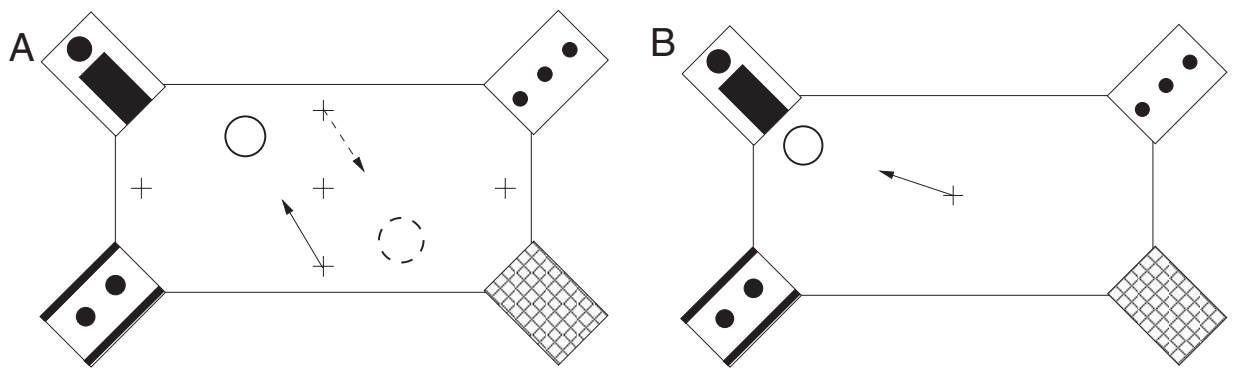

C

$\mathrm{D}$
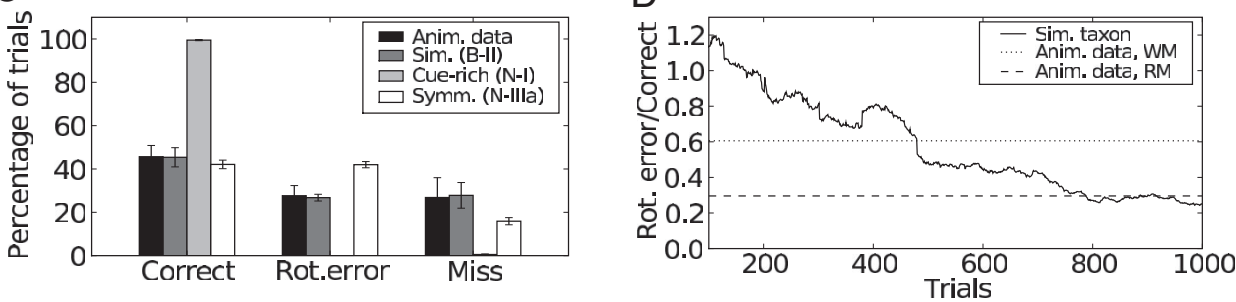

Figure 11. Experimental setup and simulation results for the experiments of Cheng (1986). A: Working memory task. The large rectangle is the environment; corners contain distinct landmarks. The small crosses mark starting positions. In the first part of a trial, rats searched for food hidden at the location marked by the solid circle (the solid arrow shows the direction to the food source from one of the staring locations, which may have been learned by the simulated rat during the learning phase). Once the food was found and partially eaten, the rat was removed, disoriented, and placed at a different starting position (e.g., near the northern wall) from which it had to find the remaining food. The rats in this experiment often made rotational errors, that is, from the new starting location, they went toward the place that was rotationally opposite to the place where the food was hidden. The dotted circle marks the location rotationally opposite to the correct food location, and the dashed arrow shows a direction of movement corresponding to the rotational error from the new starting position. Different food locations and starting positions were used in different trials. B: Reference memory task. The experimental protocol is the same as in A except that the starting position (cross) and food location (circle) remain constant from trial to trial. C: Bars show percentage of correct choices, rotational errors, and misses for real (black; data from Cheng, 1986) and simulated (dark gray; Environment B-II) rats in the experiment described in A. Animal data combine the rat choices in two versions of the working memory task (Cheng, 1986). Results of the same task performed by the simulated rats in the cue-rich $(\mathrm{N}-\mathrm{I})$ and symmetric $(\mathrm{N}-\mathrm{IIIa})$ environments are shown in light gray and white, respectively. D: Smoothed ratio of the number of rotational errors and that of correct trials during the simulation of the reference memory task described in B, as a function of the number of trials (see Method section of Simulation 3). The dotted and dashed lines show the asymptotic ratios for the real rats in the working memory and reference memory experiments, respectively (Cheng, 1986). Anim. $=$ animal; Rot. $=$ rotational; Sim. $=$ simulation; Symm. $=$ symmetric.

that rotational errors are caused in the model by the structure of visual features rather than by the arena geometry.

According to our hypothesis, a decrease in the number of rotational errors in the experiment with a fixed start (see Figure 11B) can be explained by the application of a taxon strategy. This suggestion is supported by the simulation results (see Figure 11D). In the beginning of training in the constant-start condition, the percentage of rotational errors was as high as the number of correct turns, but it gradually decreased with learning to about $30 \%$, reproducing qualitatively the results of Cheng (1986).

These results clearly show that although the visual system of the simulated rat can use landmark information to locate the goal in an egocentric search scenario, this information does not prevent the simulated rat from making rotational errors during reorientation within an allocentric frame of reference (see Figures 11C and 12). In impoverished environments, visual features from the edges of walls are often more salient than those from the landmarks, leading to rotational errors when the arrangement of landmarks is symmetric.
In addition to providing a possible explanation for the data of Cheng (1986), the simulation results are consistent with the data showing that rotational errors disappear when rats are allowed to see nonambiguous extramaze cues (Margules \& Gallistel, 1988) and that in a rectangular water maze without corner features, approximately half of the errors are rotational errors (Margules \& Gallistel, 1988; Pearce et al., 2004). Results of the experiments with rats (Cheng, 1986) and children (Hermer \& Spelke, 1996) suggested that changing the color of one of the walls does not help to decrease the number of rotational errors, in favor of the impenetrability argument mentioned above. The model is consistent with these data simply due to the fact that the visual system in the model is not sensitive to brightness and color of the visual stimuli, only to contrast. Put differently, our results suggest that edgelike visual stimuli are more salient than uniform stimuli during reorientation.

The rotational errors during navigation in the working memory task are explained in our model by rotational errors during the initial self-localization when the animal is replaced into the environment. The self-localization error is caused by the ambiguity of 
A

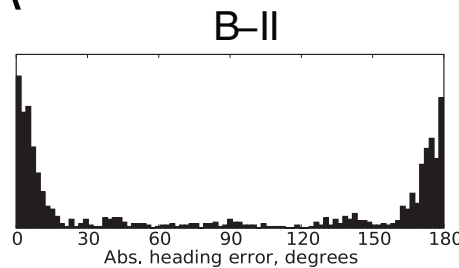

B

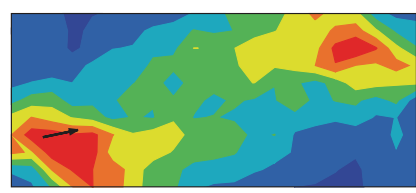

\section{C}
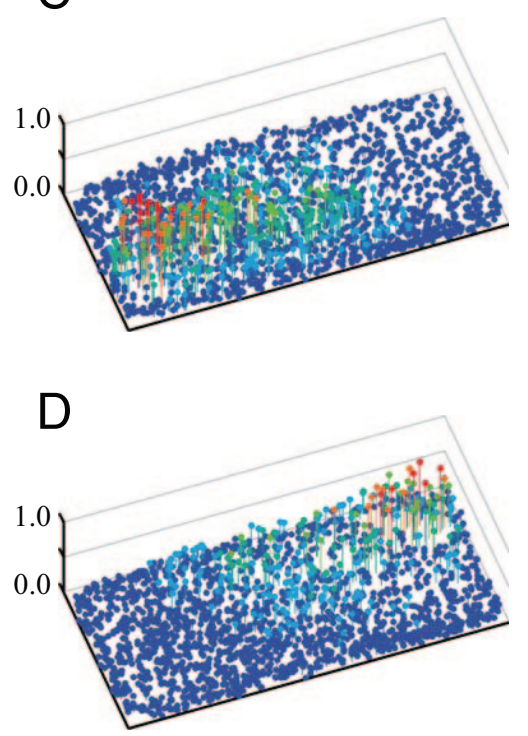
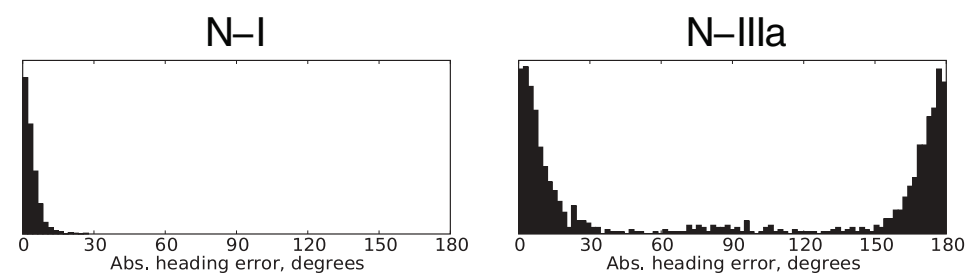

View cells: receptive fields
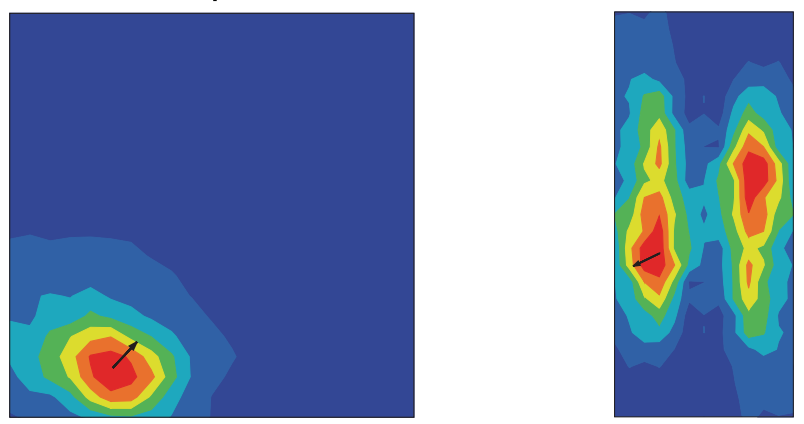

View cells: population activity
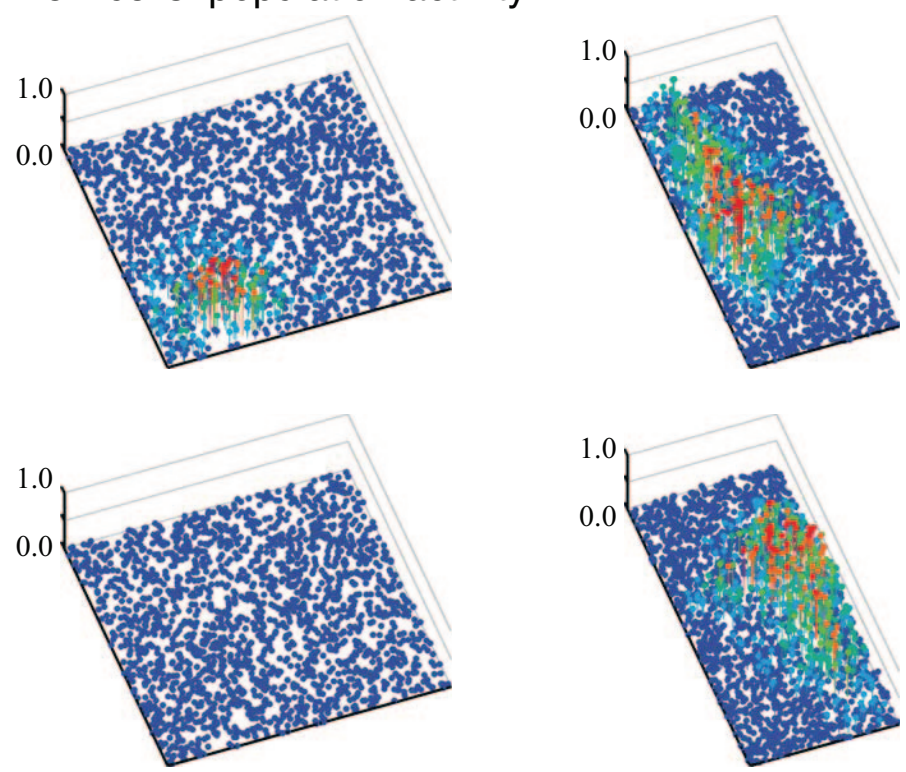

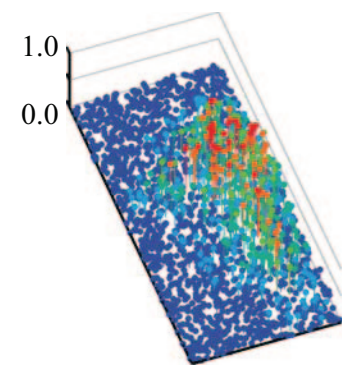

Figure 12. Allocentric coding in the view-cell population. A: Absolute values of the error in heading estimation in the simulated environment of Cheng (1986; Environment B-II, left), the cue-rich Environment N-I (middle), and the symmetric Environment N-IIIa (right) for one simulated animal. Bin size $2^{\circ}$. B: Receptive fields of example view cells in the three environments, calculated using a view-based estimation of the allocentric heading (see Method section of Simulation 3). Arrows show the position and orientation of the simulated rat at which the corresponding snapshots were taken. Note that the receptive fields of view cells are double peaked in Environments B-II (left) and N-IIIa (right) but are single peaked in the cue-rich Environment $\mathrm{N}-\mathrm{I}$ (middle). The cells in B-II and N-I are the same as those shown in Figure 3C. C, D: Activity in the population of view cells when the simulated rat is located at the position and orientation that correspond to the preferred position and orientation of the cells shown in B. Each dot represents a view cell. The position of the dot represents the location from which the snapshot was taken when the view cell was learned. The elevation of the dot from the horizontal plane and its color correspond to the activity of the cell (colors from blue to red code for activity levels from low to high). C: Population activity when the allocentric heading is estimated correctly. D: Population activity when the heading is estimated with $180^{\circ}$ error. Abs. $=$ absolute. 
visual cues that gives rise (in the case of a rectangular environment with different lengths of adjacent sides) to two different choices of directional reference. This ambiguity is resolved during head direction estimation (see Appendix, Equation A10) such that, on the level of view cells and place cells, the information about the cue ambiguity is lost and cannot be used to correct the rotational error, even after the rat fails to observe the food at the expected location. In other words, the animal does not keep a memory of its initial uncertainty. This is a direct consequence of our model approach and could be tested in experiments.

\section{General Discussion}

We have presented a model of navigation that is able to use an egocentric taxon strategy and an allocentric locale strategy to remember a goal location. The taxon strategy associates momentary views of the environment (snapshots) directly with rewarded motor actions and supports S-R behavior. The locale strategy is based on the memorized representation of the environment, which is built by associating visual snapshots with path integration in populations of grid cells and place cells. Place cells become associated with rewarded motor actions during goal learning, supporting place-based navigation.

Our model uses a view-based approach to (a) explain several key neurophysiological properties of grid cells and place cells, (b) reproduce S-R and place-based behaviors of normal and lesioned rats in the water maze, and (c) provide an explanation for key experimental results concerning the influence of environmental geometry on goal-search behavior. The view-based approach to the study of place-sensitive activity in the hippocampal formation provides an alternative to other approaches that require calculation of distances to environmental boundaries (Barry et al., 2006; Hartley, Burgess, Lever, Cacucci, \& Keefe, 2000) or landmark detection (Sharp, 1991; Touretzky \& Redish, 1996). Moreover, the view-based explanation of geometry-related effects is biologically more plausible than the explanation involving a dedicated brain module for geometry processing (Cheng, 1986; Cheng \& Newcombe, 2005; Hermer \& Spelke, 1996). These results tie together four important lines of research on animal spatial cognition: the role of place cells for behavior, the role of different navigational strategies, the role of geometry of space for spatial orientation, and the role of learning. These four aspects are now discussed in detail.

\section{Grid Cells and Place Cells}

In our model, visual input is represented exclusively by snapshots of the environment sampled by a large set of overlapping orientation-sensitive filters. Despite this simple, low-level representation, the model is able to capture a number of neurophysiological properties of grid and place cells: (a) CA1 and dMEC cells exhibit spatially localized and gridlike firing patterns, respectively (Fyhn et al., 2004; O'Keefe \& Conway, 1978); (b) anatomical topology is not observed in the CA1 population, but cells in the dMEC are organized in several subpopulations with different spatial frequencies and orientations (Hafting et al., 2005; Muller \& Kubie, 1987; O'Keefe \& Conway, 1978); (c) firing fields of both cell types rotate following a rotation of visual cues (Hafting et al., 2005; Muller \& Kubie, 1987); (d) CA1 cells stretch their fields if the environment is stretched, and some place fields disappear when the environment is shrunk (O'Keefe \& Burgess, 1996; Redish et al., 2000); and (e) firing fields of entorhinal grid cells rescale in response to stretching or shrinking of the environment (Barry et al., 2007).

Computational models of place cells (Arleo \& Gerstner, 2000; Burgess, Recce, \& O'Keefe, 1994; Hartley et al., 2000; Kali \& Dayan, 2000; Samsonovich \& McNaughton, 1997; Sharp, 1991; Touretzky \& Redish, 1996; to cite only a few) and grid cells (Burgess, Barry, \& O'Keefe, 2007; Fuhs \& Touretzky, 2006; McNaughton et al., 2006; Rolls, Stringer, \& Elliot, 2006) are numerous. In this work, we do not propose a new model of place cells or grid cells but rather try to answer the question whether the feed-forward projection hypothesis for CA1 place-cell formation is consistent with known properties of place cells, for example, dependence of place-field shapes on the geometric layout of the environment (O'Keefe \& Burgess, 1996), or their dynamics during movement along a shrinking linear track (Gothard et al., 1996). The results of our simulations suggest that the answer is positive. Several previous models of place cells have addressed the issue of place-field deformation in response to environment manipulation (Byrne et al., 2007; O'Keefe \& Burgess, 1996; Samsonovich \& McNaughton, 1997), while the phenomenon of rescaling of entorhinal firing patterns has not been considered so far, and hence, our results are novel in this respect.

The key property of place cells is that their spatial firing fields are fixed with respect to the external environment, making it possible to treat their activity as a location signal. In our model, this property is mainly due to the connections from view cells (via grid cells) to place cells. The activity of a view cell depends strongly on the current allocentric location of the animal and only weakly on the current allocentric orientation (see Figure 3), such that a population of view cells can reliably fix the place field in the environment. Hartley et al. (2000) put forward a model of placecell firing in which boundary vector cells (BVCs) play a role similar to that of view cells in our model. A BVC responds maximally when the set of distances to currently observed boundaries matches those to which the BVC is tuned. Consider an environment in which the only visual cues are formed by the edges of a wall (i.e., no visual pattern is present on the wall, the floor, or the ceiling of the environment). Under the assumption that the current allocentric heading is estimated correctly, a view cell that stores a snapshot in direction $\Phi$ in this environment would be equivalent to a BVC with preferred direction $\Phi$. The preferred distance of the BVC would be encoded in the position of the wall edges on the snapshot image.

However, despite their equivalence in some cases, view cells are conceptually different from BVCs, since distance calculation and image matching are quite different operations. The difference is immediately seen if, in our example, the height of the wall is changed after the environment has been explored. The change in the wall height would cause view cells in our model to fire in a different location, defined by view matching. In a navigation task, such a change would cause the animal to search for a goal in a different position, with respect to the environment where the height of the wall remained the same. No such change would be observed in the BVC model. To our knowledge, there is no experimental data on rats that can provide direct support for one or the other model. For species other than rats (e.g., honeybees and pigeons), experimental evidence has suggested that visual infor- 
mation is treated differently depending on the species (K. Cheng, personal communication, November 3, 2008).

The model of place-cell firing described here includes visual input and path integration and does not take into account the potential role of olfactory and tactile information. This is a clear limitation of the present model and has to be addressed in future work.

\section{Taxon and Locale Strategies}

On the behavioral level, the model reproduces rat behavior in variable- and constant-start versions of the water-maze task and is consistent with a number of lesion studies. In a common view, locale strategies involve cognitive mapping abilities that allow the animal to compute its path (i.e., perform some sort of trajectory planning) toward the place where the goal was encountered previously (Morris, 1981; O'Keefe \& Nadel, 1978). This is usually put in contrast to $\mathrm{S}-\mathrm{R}$ learning, which associates motor responses to relevant stimuli using Pavlovian-like conditioning mechanisms (Devan \& White, 1999; Packard \& McGaugh, 1996).

In our model, both taxon and locale strategies learn to approach the goal using the same reward-based algorithm that associates stimuli with motor responses, and hence, both can be considered as $\mathrm{S}-\mathrm{R}$-based strategies, in contradiction to the common view. The distinction between the two strategies in our model lies in the fact that the notion of stimulus $\mathrm{S}$ is interpreted differently for the two strategies. In the case of taxon strategy, the stimuli are directly encoded as visual features and become associated with motor actions during reward-based learning. In contrast, the locale strategy is learned in two phases: First, the visual features are processed to yield place-cell activities in a phase of unrewarded latent learning; second, the place-cell activities are used as stimuli during the S-R learning phase.

Despite the fact that the locale strategy in the model is based on S-R learning and does not involve trajectory planning, the behavior of the simulated rat in the Morris water-maze task is remarkably similar to that of real rats. This suggests that for wide range of navigation tasks in which an animal has to remember the position of a hidden goal in a fixed environment, the navigation strategy that is often termed cognitive or map-based can be implemented by a simple, associative learning mechanism (Chamizo, 2003), based on place cells. It does not exclude, of course, that true planning abilities may be needed in other spatial tasks, such as those that require making shortcuts.

\section{Influence of Environmental Geometry}

An important conclusion from our results concerns the effects of environmental geometry on the activity of spatially selective cells and goal-oriented behavior. Our results suggest that the influence of geometry of space observed in experimental data is a by-product of visual information processing. Rotational errors, observed during reorientation in rectangular arenas, are caused in the model by the structure of visual inputs, rather than by the arena geometry. Hence, we argue that the concept of a geometric module is not necessary. More precisely, (a) if the geometric module is viewed as a separate brain structure responsible for geometry-related calculations, then there is no need of such a structure, since our model can reproduce Cheng's (1986) results without it; and (b) if the geometric module is meant to be a theoretical abstraction, then we question the explanatory power of this abstraction. In environments in which the arrangement of walls is symmetric, the edges of walls represent ambiguous cues, whereas visual patterns attached to the walls (e.g., landmarks) represent nonambiguous cues. Behavioral decisions made on the basis of the ambiguous cues may appear to be caused by the arena geometry but could in fact be based on sets of local features arranged in a symmetric and hence ambiguous configuration. The latter seems to us to be a more parsimonious explanation.

The idea that a simple navigation strategy based on view-based matching may explain the rotation errors in the experiment of Cheng (1986) was very recently investigated by Stürzl et al. (2008) in parallel to our own work (Sheynikhovich, 2007). They showed that a simple snapshot-matching navigation strategy can explain rotational errors in a quasi-symmetric environment similar to the one used in the experiment of Cheng. Apart from proposing the link between the orientation errors and activities of place cells and grid cells in the hippocampal formation, our present model extends their findings in two important ways. First, our model proposes a biologically plausible mechanism of reward-based learning of navigational strategies, whereas, in the standard view-based matching algorithms, the navigating animal moves in such a way as to increase the match between the currently visible snapshot and the a priori known target snapshot (Collett \& Collett, 2002; Stürzl et al., 2008). Second, due to the learning of two different strategies in the model, we were able to account for the persistence of rotational errors during working memory experiment, and for the decrease of the errors during the reference memory experiment of Cheng. Although, in both cases, the rotational errors were caused by the ambiguity in the visual input, there is a fundamental difference in the way the two navigational strategies deal with this ambiguity in the model. During reorientation required by the locale strategy, rotational errors result from the matching process between the currently perceived snapshots and all snapshots stored in memory during exploration. Since snapshots are taken in random directions, the ambiguity of visual cues and the number of rotational errors do not decrease with reward-based training and are independent of the starting location. The situation is different for the taxon strategy that associates incoming snapshots with rewarded rotation angles. In the beginning of training in the constant-start condition, the number of snapshots that are already associated with turns by the correct angle is low, and the number of rotational errors is high (see Figure 11D). However, as the learning continues, progressively more snapshots become associated with corresponding rotations, leading to the decrease of rotational errors with training. In other words, the model suggests that the decrease in the number of rotational errors when switching from variable to constant starting positions might be due to change in navigational strategy.

We would like to emphasize that although, in our simulations, the effect of geometry was due to the presence of opaque walls surrounding the arena, the wall presence is not a necessary condition for the model to work. Figure 13 illustrates this point. In Figure 13A (top), we show a snapshot taken from the center of the simulated water-maze environment, with a gray circular wall, white water, and multiple background cues. Even if the wall is made absolutely transparent (see Figure 13B, top), the edge formed by the surface of the maze and the background can still be 
A
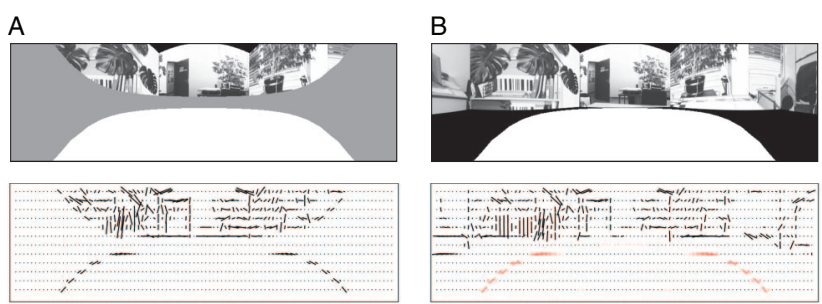

Figure 13. Visual input in the model in the case of transparent walls or an elevated environment without walls. Top row: Snapshots taken from the center of a simulated environment consisting of a circular arena with white surface and opaque gray walls (A) or invisible walls (B), located inside of a large square room with multiple visual cues and black floor. Bottom row: Filter representation of the two snapshots. Even in the absence of the opaque wall, its border with the white arena surface can be detected by the visual system (filter responses corresponding to the surface edge in the environment with invisible walls are shown in red).

visible (unless special precautions are taken to diminish the visibility in the experiment) and can be used as a cue. The same holds true for the case of the edge formed by a drop (i.e., when an elevated experimental arena has no walls; Barry et al., 2006). The bottom panels in Figure 13 show the filter responses to the corresponding snapshots. In an environment with a lot of polarizing background cues, the filter responses to the edge of the arena surface might not play an important role, whereas, in a visually impoverished environment, they might provide information about the position of the animal with respect to the maze edge (which might be interpreted as encoding of the distance to the transparent wall, Maurer \& Derivaz, 2000, or to the drop, Barry et al., 2006).

\section{Latent Unsupervised Learning Versus Reward-Based Learning}

In our model, visual input is encoded by an ensemble of view cells. Each view cell has learned and stored a particular view of the environment during exploration. The learning and recruitment occur in an unsupervised manner and are independent of reward. Similarly, the connections between view cells and grid cells and those between grid cells and place cells are set according to an unsupervised Hebbian learning rule. Hence, the representation of the environment by place cells is formed in a completely unsupervised manner, that is, independent of the reward structure, akin to the concept of latent learning in psychology.

Given the representation by place cells, the model learns to perform the appropriate actions to reach the escape platform using locale navigation strategy. The learning of the locale strategy is triggered by reward given at the target location. Hence, the locale pathway in our model has a preprocessing stream from visual input to place cells that is independent of reward and formed by latent unsupervised learning. Preprocessing is followed by the association between places and actions learned by reward-modulated plasticity. The taxon strategy in our model is, as a whole, reward dependent, since the association between visual input and actions is learned by reinforcement learning.

We speculate that the differences in learning between the two pathways may partially explain recent experiments of blocking and overshadowing effects that show a difference between local in- tramaze landmarks and global environmental shape on goal-search behavior (Doeller \& Burgess, 2008; Hayward, Good, \& Pearce, 2004). In a pure reward-based learning paradigm, if one stimulus is learned to reliably predict reinforcement, it will prevent (or block) learning of associations between other stimuli and the reinforcement. For example, if a visual cue present in the view field predicts that turning by an angle $\theta$ will result in reward (e.g., by strengthening connection weights between the visual filters corresponding to the position of the cue in the snapshot and the action cell corresponding to turning angle $\theta$ ), then adding a second visual cue will not give rise to a weight increase between the second cue and (the same) action cell (resulting from the fact that $\delta$ in Equation A4 in the Appendix will be equal to 0 after the association between the first cue and reward has been learned). Thus, since taxon navigation in our model is based on pure S-R association learned by a reinforcement learning rule, taxon navigation should show overshadowing or blocking. Consequently, an object that was learned to be consistently located with respect to a first landmark cannot be located with respect to a second landmark that is added on, or made consistent, only later (Doeller \& Burgess, 2008).

For the locale strategy, however, the situation is different. If a sufficiently salient cue (i.e., the one that triggers creation of new view cells) is added after an object location has already been learned by the locale strategy, the new view cells might become associated by unsupervised Hebbian learning with grid cells and place cells in the initial (reward-independent) processing stream from visual input to place cells. These new view cells will then be able to drive behavior (via the connections to grid cells and place cells to action cells) even in the absence of the previously learned cues. This might explain why learning an object location with respect to one part of the wall does not block learning with respect to another part of the wall in a circular maze (Doeller \& Burgess, 2008). In our simulations, a part of the wall constitutes a prominent visual cue and hence could potentially be encoded by new view cells that are formed independent of reward.

Finally, the model assumptions that the taxon and locale strategies can be learned in parallel and are mediated by separate memory systems suggest an explanation for the absence of blocking and overshadowing between intramaze landmarks (that allow taxon learning) and the shape of the environment (that favors place-based learning; Hayward et al., 2004; Hayward, McGregor, Good, \& Pearce, 2003). However, a detailed model of interaction between the two strategies (Chavarriaga, Strösslin, Sheynikhovich, \& Gerstner, 2005a, 2005b) is required to explain the precise pattern of overshadowing and blocking effects in various cue configurations and training protocols (Doeller \& Burgess, 2008; Roberts \& Pearce, 1998), which is out of the scope of the present article.

\section{Predictions Derived From the Model}

Several predictions can be made in relation to our results concerning the issue of the effect of geometry on place-cell firing and behavior. First, since rotational errors are mainly caused by the arrangement of visual features in the environment, their number can be decreased either by making the overall arrangement of landmarks nonsymmetric or by making the landmarks sufficiently different. For example, adding a disambiguating visual feature at 
the middle of one of the walls should decrease rotational error in the working memory experiment of Cheng (1986). This prediction is supported by the data from reorientation experiments with children, in which rotational errors decreased significantly when a bookshelf was placed at the middle of one of the walls of the rectangular testing room (Learmonth, Newcombe, \& Huttenlocher, 2001). Second, since the decrease in the number of rotational errors is explained in the model by strategy switching, we predict that lesioning the taxon pathway $(\mathrm{CP}$ or $\mathrm{SNc})$ will increase the number of rotational errors in the reference memory task (see Figure 11B) relative to controls, while not changing the performance in the working memory task (see Figure 11A). Third, since, in our simulations, the variability of starting position strongly biased the simulated rat toward using the locale strategy, we predict that changing starting position from trial to trial in the reference memory experiment of Cheng would result in an increase in the number of rotational errors, with respect to the case of fixed starting position. Finally, the model predicts that when a rotational error is committed, place cells (and grid cells) corresponding to the location that is rotationally opposite to the actual one should be active (Lenck-Santini, Muller, Save, \& Poucet, 2002; O'Keefe \& Speakman, 1987). In other words, the rat thinks it is at a different, diagonally opposite place, rather than at the place where it actually is.

\section{References}

Annett, L. E., McGregor, A., \& Robbins, T. W. (1989). The effects of ibotenic acid lesions of the nucleus accumbens on spatial learning and extinction in the rat. Behavioural Brain Research, 31, 231-242.

Arleo, A., \& Gerstner, W. (2000). Spatial cognition and neuro-mimetic navigation: A model of hippocampal place cell activity. Biological Cybernetics, 83, 287-299.

Arleo, A., \& Gerstner, W. (2001). Spatial orientation in navigating agents: Modeling head-direction cells. Neurocomputing, 38-40, 1059-1065.

Barry, C., Hayman, R., Burgess, N., \& Jeffery, K. J. (2007). Experiencedependent rescaling of entorhinal grids. Nature Neuroscience, 10, 682684

Barry, C., Lever, C., Hayman, R., Hartley, T., Burton, S., O’Keefe, J., et al. (2006). The boundary vector cell model of place cell firing and spatial memory. Reviews in the Neurosciences, 17, 71-97.

Biegler, R., \& Morris, R. G. M. (1993, February 18). Landmark stability is a prerequisite for spatial but not discrimination learning. Nature, 361, 631-633.

Brown, M. A., \& Sharp, P. E. (1995). Simulation of spatial learning in the Morris water maze by a neural network model of the hippocampalformation and nucleus accumbens. Hippocampus, 5, 171-188.

Brun, V. H., Otnass, M. K., Molden, S., Steffenach, H. A., Witter, M. P., Moser, M. B., et al. (2002, June 21). Place cells and place recognition maintained by direct entorhinal-hippocampal circuitry. Science, 296, 2243-2246.

Burgess, N., Barry, C., \& O'Keefe, J. (2007). An oscillatory interference model of grid cell firing. Hippocampus, 17, 801-812.

Burgess, N., Recce, M., \& O'Keefe, J. (1994). A model of hippocampal function. Neural Networks, 7, 1065-1081.

Byrne, P., Becker, S., \& Burgess, N. (2007). Remembering the past and imagining the future: A neural model of spatial memory and imagery. Psychological Review, 114, 340-375.

Cartwright, B., \& Collett, T. (1982, August 5). How honey bees use landmarks to guide their return to a food source. Nature, 295, 560-564.

Cartwright, B., \& Collett, T. (1983). Landmark learning in bees. Journal of Comparative Physiology: Sensory, Neural, and Behavioral Physiology, 151(A), 521-543.
Cash, S., \& Yuste, R. (1999). Linear summation of excitatory inputs by CA1 pyramidal neurons. Neuron, 22, 383-394.

Chamizo, V. D. (2003). Acquisition of knowledge about spatial location: Assessing the generality of the mechanism of learning. Quarterly Journal of Experimental Psychology: Comparative and Physiological Psychology, 56(B), 102-113.

Chavarriaga, R., Strösslin, T., Sheynikhovich, D., \& Gerstner, W. (2005a). Competition between cue response and place response: A model of rat navigation behaviour. Connection Science, 17, 167-183.

Chavarriaga, R., Strösslin, T., Sheynikhovich, D., \& Gerstner, W. (2005b). A computational model of parallel navigation systems in rodents. Neuroinformatics, 3, 223-242.

Cheng, K. (1986). A purely geometric module in the rat's spatial representation. Cognition, 23, 149-178.

Cheng, K., \& Newcombe, N. S. (2005). Is there a geometric module for spatial orientation? Squaring theory and evidence. Psychonomic Bulletin \& Review, 12, 1-23.

Cheung, A., Stürzl, W., Zeil, J., \& Cheng, K. (2008). The information content of panoramic images: II. The rotational errors and the similarity of views in rectangular experimental arenas. Journal of Experimental Psychology: Animal Behavior Processes, 34, 15-30.

Collett, T. S., \& Collett, M. (2002). Memory use in insect visual navigation. Nature Reviews Neuroscience, 3, 542-552.

Cressant, A., Muller, R. U., \& Poucet, B. (1997). Failure of centrally placed objects to control firing fields of hippocampal place cells. Journal of Neuroscience, 17, 2531-2542.

Cressant, A., Muller, R. U., \& Poucet, B. (1999). Further study of the control of place cell firing by intra-apparatus objects. Hippocampus, 9 , 423-431.

Da Cunha, C., Silva, M. H., Wietzikoski, S., Wietzikoski, E. C., Ferro, M. M., Kouzmine, I., et al. (2006). Place learning strategy of substantia nigra pars compacta-lesioned rats. Behavioural Neuroscience, 120, $1279-1284$.

Da Cunha, C., Wietzikoski, S., Wietzikoski, E. C., Miyoshi, E., Ferro, M. M., Anselmo-Franci, J. A., et al. (2003). Evidence for the substantia nigra pars compacta as an essential component of a memory system independent of the hippocampal memory system. Neurobiology of Learning and Memory, 79, 236-242.

Deneve, S., Latham, P. E., \& Pouget, A. (1999). Reading population codes: A neural implementation of ideal observers. Nature Neuroscience, 2, $740-745$.

Deneve, S., Latham, P. E., \& Pouget, A. (2001). Efficient computation and cue integration with noisy population codes. Nature Neuroscience, 4 , $826-831$

Devan, B. D., \& White, N. M. (1999). Parallel information processing in the dorsal striatum: Relation to hippocampal function. Journal of Neuroscience, 19, 2789-2798.

Doeller, C. F., \& Burgess, N. (2008). Distinct error-correcting and incidental learning of location relative to landmarks and boundaries. Proceedings of the National Academy of Sciences, USA, 105, 5909-5914.

Eichenbaum, H., Stewart, C., \& Morris, R. G. (1990). Hippocampal representation in place learning. Journal of Neuroscience, 10, 3531-3542.

Epstein, R., Graham, K. S., \& Downing, P. E. (2003). Viewpoint-specific scene representations in human parahippocampal cortex. Neuron, 37, 865-876.

Etienne, A. S., \& Jeffery, K. J. (2004). Path integration in mammals. Hippocampus, 14, 180-192.

Fiete, I. R., Burak, Y., \& Brookings, T. (2008). What grid cells convey about rat location. Journal of Neuroscience, 28, 6858-6871.

Fodor, J. A. (1983). The modularity of mind. Cambridge, MA: MIT Press.

Foley, J. D., van Dam, A., Feiner, S. K., \& Hughes, J. F. (1995). Computer graphics: Principles and practice. Reading, MA: Addison-Wesley.

Franz, M. O., Schölkopf, B., Mallot, H. A., \& Bülthoff, H. H. (1998). 
Where did I take that snapshot? Scene-based homing by image matching. Biological Cybernetics, 79, 191-202.

Fuhs, M. C., \& Touretzky, D. S. (2006). A spin glass model of path integration in rat medial entorhinal cortex. Journal of Neuroscience, 26, $4266-4276$.

Fyhn, M., Molden, S., Witter, M. P., Moser, E. I., \& Moser, M. B. (2004, August 27). Spatial representation in the entorhinal cortex. Science, 305, $1258-1264$.

Gallistel, C. R. (1990). The organization of learning. Cambridge, MA: MIT Press.

Gasparini, S., \& Magee, J. C. (2006). State-dependent dendritic computation in hippocampal CA1 pyramidal neurons. Journal of Neuroscience, 26, 2088-2100.

Gaunet, F., Vidal, M., Kemeny, A., \& Berthoz, A. (2001). Active, passive and snapshot exploration in a virtual environment: Influence on scene memory, reorientation and path memory. Cognitive Brain Research, 11, $409-420$.

Gillner, S., \& Mallot, H. A. (1998). Navigation and acquisition of spatial knowledge in a virtual maze. Journal of Cognitive Neuroscience, 10, 445-463.

Girman, S. V., Sauvé, Y., \& Lund, R. D. (1999). Receptive field properties of single neurons in rat primary visual cortex. Journal of Neurophysiology, 82, 301-311.

Gothard, K. M., Hoffman, K. L., Battaglia, F. P., \& McNaughton, B. L. (2001). Dentate gyrus and CA1 ensemble activity during spatial reference frame shifts in the presence and absence of visual input. Journal of Neuroscience, 21, 7284-7292.

Gothard, K. M., Skaggs, W. E., \& McNaughton, B. L. (1996). Dynamics of mismatch correction in the hippocampal ensemble code for space: Interaction between path integration and environmental cues. Journal of Neuroscience, 16, 8027-8040.

Hafting, T., Fyhn, M., Molden, S., Moser, M. B., \& Moser, E. I. (2005, August 11). Microstructure of a spatial map in the entorhinal cortex. Nature, 436, 801-806.

Hartley, T., Burgess, N., Lever, C., Cacucci, F., \& Keefe, J. O. (2000). Modeling place fields in terms of the cortical inputs to the hippocampus. Hippocampus, 10, 369-379.

Hayward, A., Good, M. A., \& Pearce, J. M. (2004). Failure of a landmark to restrict spatial learning based on the shape of the environment. Quarterly Journal of Experimental Psychology: Comparative and Physiological Psychology, 57(B), 289-314.

Hayward, A., McGregor, A., Good, M. A., \& Pearce, J. M. (2003). Absence of overshadowing and blocking between landmarks and the geometric cues provided by the shape of a test arena. Quarterly Journal of Experimental Psychology: Comparative and Physiological Psychology, 56(B), 114-126.

Hermer, L., \& Spelke, E. (1996). Modularity and development: The case of spatial reorientation. Cognition, 61, 195-232.

Hill, A. J. (1978). First occurrence of hippocampal spatial firing in a new environment. Experimental Neurology, 62, 282-297.

Hull, C. L. (1943). Principles of behavior: An introduction to behavior theory. New York: Appleton-Century.

Kali, S., \& Dayan, P. (2000). The involvement of recurrent connections in area CA3 in establishing the properties of place fields: A model. Journal of Neuroscience, 20, 7463-7477.

Kelley, A. E., \& Domesick, V. B. (1982). The distribution of the projection from the hippocampal formation to the nucleus accumbens in the rat: An anterograde- and retrograde-horseradish peroxidase study. Neuroscience, 7, 2321-2335.

Kolb, B., \& Tees, R. C. (Eds.). (1990). The cerebral cortex of the rat. Cambridge, MA: MIT Press.

Learmonth, A. E., Newcombe, N. S., \& Huttenlocher, J. (2001). Toddlers' use of metric information and landmarks to reorient. Journal of Experimental Child Psychology, 80, 225-244.
Lenck-Santini, P.-P., Muller, R. U., Save, E., \& Poucet, B. (2002). Relationships between place cell firing fields and navigational decisions by rats. Journal of Neuroscience, 22, 9035-9047.

Maaswinkel, H., \& Whishaw, I. Q. (1999). Homing with locale, taxon, and dead reckoning strategies by foraging rats: Sensory hierarchy in spatial navigation. Behavioural Brain Research, 99, 143-152.

Margules, J., \& Gallistel, C. R. (1988). Heading in the rat: Determination by environmental shape. Animal Learning \& Behavior, 16, 404-410.

Maurer, R., \& Derivaz, V. (2000). Rats in a transparent Morris water maze use elemental and configural geometry of landmarks as well as distance to the pool wall. Spatial Cognition and Computation, 2, 135-156.

McDonald, R. J., Hong, N. S., \& Devan, B. D. (2004). The challenges of understanding mammalian cognition and memory-based behaviours: An interactive learning and memory systems approach. Neuroscience and Biobehavioral Reviews, 28, 719-745.

McNaughton, B. L., Battaglia, F. P., Jensen, O., Moser, E. I., \& Moser, M. B. (2006). Path integration and the neural basis of the "cognitive map." Nature Reviews Neuroscience, 7, 663-678.

Mizumori, S. J. Y., \& Williams, J. D. (1993). Directionally selective mnemonic properties of neurons in the lateral dorsal nucleus of the thalamus of rats. Journal of Neuroscience, 13, 4015-4028.

Morris, R. G. M. (1981). Spatial localization does not require the presence of local cues. Learning and Motivation, 12, 239-260.

Morris, R. G. M., Garrud, P., Rawlins, J. N., \& O'Keefe, J. (1982, June 24). Place navigation impaired in rats with hippocampal lesions. Nature, 297, 681-683.

Muller, R. U., \& Kubie, J. L. (1987). The effects of changes in the environment on the spatial firing of hippocampal complex-spike cells. Journal of Neuroscience, 7, 1951-1968.

Muller, R. U., Kubie, J. L., \& Ranck, J. J. B. (1987). Spatial firing patterns of hippocampal complex-spike cells in a fixed environment. Journal of Neuroscience, 7, 1935-1950.

Oja, E. (1982). Simplified neuron model as a principal component analyzer. Journal of Mathematical Biology, 15, 267-273.

O'Keefe, J., \& Burgess, N. (1996, May 30). Geometric determinants of the place fields of hippocampal neurons. Nature, 381, 425-428.

O'Keefe, J., \& Burgess, N. (2005). Dual phase and rate coding in hippocampal place cells: Theoretical significance and relationship to entorhinal grid cells. Hippocampus, 15, 853-866.

O'Keefe, J., \& Conway, D. H. (1978). Hippocampal place units in the freely moving rat: Why they fire where they fire. Experimental Brain Research, 31, 573-590.

O'Keefe, J., \& Dostrovsky, J. (1971). The hippocampus as a spatial map. Preliminary evidence from unit activity in the freely-moving rat. Brain Research, 34, 171-175.

O'Keefe, J., \& Nadel, L. (1978). The hippocampus as a cognitive map. Oxford, England: Clarendon Press.

O'Keefe, J., \& Speakman, A. (1987). Single unit activity in the rat hippocampus during a spatial memory task. Experimental Brain Research, 68, 1-27.

Packard, M. G., \& McGaugh, J. L. (1992). Double dissociation of fornix and caudate nucleus lesions on acquisition of two water maze tasks: Further evidence for multiple memory systems. Behavioural Neuroscience, 106, 439-446.

Packard, M. G., \& McGaugh, J. L. (1996). Inactivation of hippocampus or caudate nucleus with lidocaine differentially affects expression of place and response learning. Neurobiology of Learning and Memory, 65, $65-72$.

Pawlak, V., \& Kerr, J. N. (2008). Dopamine receptor activation is required for corticostriatal spike-timing-dependent plasticity. Journal of Neuroscience, 28, 2435-2446.

Pearce, J. M., Good, M. A., Jones, P. M., \& McGregor, A. (2004). Transfer of spatial behavior between different environments: Implications for theories of spatial learning and for the role of the hippocampus in spatial 
learning. Journal of Experimental Psychology: Animal Behavior Processes, 30, 135-147.

Poucet, B., Lenck-Santini, P.-P., \& Save, E. (2003). Drawing parallels between the behavioural and neural properties of navigation. In K. J. Jeffery (Ed.), The neurobiology of spatial behaviour (pp. 187-198). Oxford, England: Oxford University Press.

Quirk, G. J., Muller, R. U., \& Kubie, J. L. (1990). The firing of hippocampal place cells in the dark depends on the rat's recent experience. Journal of Neuroscience, 10, 2008-2017.

Ranck, J. B., Jr. (1984). Head-direction cells in the deep cell layers of dorsal presubiculum in freely moving rats. Society for Neuroscience Abstracts, 10, 599.

Redish, A. D. (1999). Beyond the cognitive map: From place cells to episodic memory. London: Bradford Books.

Redish, A. D., Rosenzweig, E. S., Bohanick, J. D., McNaughton, B. L., \& Barnes, C. A. (2000). Dynamics of hippocampal ensemble activity realignment: Time versus space. Journal of Neuroscience, 20, 9298 9309.

Roberts, A. D., \& Pearce, J. M. (1998). Control of spatial behavior by an unstable landmark. Journal of Experimental Psychology: Animal Behavior Processes, 24, 172-184.

Rolls, E. T., Stringer, S. M., \& Elliot, T. (2006). Entorhinal cortex grid cells can map to hippocampal place cells by competitive learning. Network: Computation in Neural Systems, 17, 447-465.

Samsonovich, A., \& McNaughton, B. L. (1997). Path integration and cognitive mapping in a continuous attractor neural network model. Journal of Neuroscience, 17, 5900-5920.

Save, E., Nerad, L., \& Poucet, B. (2000). Contribution of multiple sensory information to place field stability in hippocampal place cells. Hippocampus, 10, 64-76.

Schultz, W. (1998). Predictive reward signal of dopamine neurons. Journal of Neurophysiology, 80, 1-27.

Schultz, W., Dayan, P., \& Montague, P. R. (1997, March 14). A neural substrate of prediction and reward. Science, 275, 1593-1599.

Sesack, S. R., \& Pickel, V. M. (1990). In the rat medial nucleus accumbens, hippocampal and catecholaminergic terminals converge on spiny neurons and are in apposition to each other. Brain Research, 527, 266-279.

Sharp, P. E. (1991). Computer simulation of hippocampal place cells. Psychobiology, 19, 103-115.

Sheynikhovich, D. (2007). Spatial navigation in geometric mazes: A computational model of rodent behavior. Unpublished doctoral thesis, École Polytechnique Fédérale de Lausanne, Lausanne, Switzerland.

Skaggs, W. E., Knierim, J. J., Kudrimoti, H. S., \& McNaughton, B. L. (1995). A model of the neural basis of the rat's sense of direction. In G. Tesauro, D. S. Touretzky, \& T. K. Leen (Eds.), Advances in neural information processing systems (Vol. 7, pp. 173-180). Cambridge, MA: MIT Press.
Solstad, T., Moser, E. I., \& Einevoll, G. T. (2006). From grid cells to place cells: A mathematical model. Hippocampus, 16, 1026-1031.

Spiers, H. J., Burgess, N., Hartley, T., Vargha-Khadem, F., \& O'Keefe, J. (2001). Bilateral hippocampal pathology impairs topographical and episodic memory but not visual pattern matching. Hippocampus, 11, 715725

Strösslin, T., Sheynikhovich, D., Chavarriaga, R., \& Gerstner, W. (2005). Robust self-localisation and navigation based on hippocampal place cells. Neural Networks, 8, 1125-1140.

Stürzl, W., Cheung, A., Cheng, K., \& Zeil, J. (2008). The information content of panoramic images: I. The rotational errors and the similarity of views in rectangular experimental arenas. Journal of Experimental Psychology: Animal Behavior Processes, 34, 1-14.

Sutherland, R. J., \& Rodriguez, A. J. (1990). The role of the fornix/fimbria and some related subcortical structures in place learning and memory. Behavioural Brain Research, 32, 265-277.

Sutton, R., \& Barto, A. G. (1998). Reinforcement learning: An introduction. Cambridge, MA: MIT Press.

Suzuki, S., Augerinos, G., \& Black, A. (1980). Stimulus control of spatial behavior on the eight-arm maze in rats. Learning and Motivation, 11, $1-18$.

Taube, J. S., Muller, R. U., \& Ranck, J. B. (1990a). Head-direction cells recorded from the postsubiculum in freely moving rats: I. Description and quantitative analysis. Journal of Neuroscience, 10, 420-435.

Taube, J. S., Muller, R. U., \& Ranck, J. B. (1990b). Head-direction cells recorded from the postsubiculum in freely moving rats: II. Effects of environmental manipulations. Journal of Neuroscience, 10, 436-447.

Tolman, E. C. (1948). Cognitive maps in rats and men. Psychological Review, 55, 189-208.

Touretzky, D. S., \& Redish, A. D. (1996). A theory of rodent navigation based on interacting representations of space. Hippocampus, 6, 247-270.

Wang, R., \& Spelke, E. (2002). Human spatial representation: Insights from animals. Trends in Cognitive Sciences, 6, 376-382.

Wang, R., \& Spelke, E. (2003). Comparative approaches to human navigation. In K. J. Jeffery (Ed.), The neurobiology of spatial behaviour (pp. 119-143). Oxford, England: Oxford University Press.

Whishaw, I. Q., Hines, D. J., \& Wallace, D. G. (2001). Dead reckoning (path integration) requires the hippocampal formation: Evidence from spontaneous exploration and spatial learning tasks in light (allothetic) and dark (idiothetic) tests. Behavioural Brain Research, 127, 49-69.

Whishaw, I. Q., \& Maaswinkel, H. (1997). Absence of dead reckoning in hippocampal rats. Society for Neuroscience Abstracts, 23, 1839.

White, N. M., \& McDonald, R. J. (2002). Multiple parallel memory systems in the brain of the rat. Neurobiology of Learning and Memory, $77,125-184$.

Wilson, M. A., \& McNaughton, B. L. (1993, August 20). Dynamics of the hippocampal ensemble code for space. Science, 261, 1055-1058. 


\section{Appendix}

\section{Implementation of the Model}

In all simulations, the position of the model rat changed in discrete time steps $\Delta t=0.125 \mathrm{~s}$. The running speed $v$ of the model rat was constant and equal to $16 \mathrm{~cm} / \mathrm{s}$. The values of model parameters used in the equations below are listed in Table A1.

\section{Visual Input}

An example of a two-dimensional Gabor filter sensitive to vertical lines in the image is shown in Figure 3B, left (inset), in the main text. Such a Gabor filter is a two-dimensional complex wavelet defined in the space domain as

$$
g\left(\vec{x}_{k l}, \vec{w}_{m}\right)=\exp \left(-\frac{\left\|\vec{x}-\vec{x}_{k l}\right\|^{2}}{2 \sigma_{g}^{2}}\right) \cdot \exp \left(\mathrm{i} \vec{w}_{m} \cdot\left(\vec{x}-\vec{x}_{k l}\right)\right),
$$

where $\vec{x}_{k l}=\left(x_{k l}, y_{k l}\right)$ is the coordinate of the grid point $(k, l)$ at which the filter is centered in visual space, $\vec{w}_{m} /\left\|\vec{w}_{m}\right\|$ defines the filter orientation, $\left\|\vec{w}_{m}\right\| / 2 \pi$ is the frequency of the modulating sinusoidal wave, $\sigma_{g}$ is the width of the circular receptive field, and $\vec{x}$ is running over all pixels in the image. Sampling in our model of visual input is sufficiently dense so that the distance between the nearby grid points is $2 \sigma_{g}$. A response of the filter to the corresponding portion of the gray-level image $I$ perceived at time $t$ is characterized by its amplitude:

$$
r_{k l m}^{\mathrm{vis}}(t)=\sqrt{\left(\Re\left[g\left(\vec{x}_{k l}, \vec{w}_{m}\right)\right] * I(t)\right)^{2}+\left(\mathfrak{I}\left[g\left(\vec{x}_{k l}, \vec{w}_{m}\right)\right] * I(t)\right)^{2}},
$$

where $\mathfrak{R}[\cdot]$ and $\mathfrak{I}[\cdot]$ are the real and imaginary parts, respectively, and $\langle *\rangle$ denotes integration over visual space. The set of $K=k \times l \times m$ filter amplitudes $r_{j}^{\text {vis }}$ where index $j$ runs over all grid points and orientations, serves as the internal neural representation of the visual snapshot observed at time $t$.

\section{Learning of Taxon and Locale Strategies}

Action cell $i$ in the model of caudate-putamen (CP) represents a particular direction of movement $\psi_{i}=2 \pi i / N_{\mathrm{ac}}$, where $N_{\mathrm{ac}}=360$ cells. The action cells are driven by the responses of visual filters to input snapshots, such that the activity of a cell $i$ is equal to a weighted sum of the presynaptic input $r_{i}^{\mathrm{CP}}=\sum_{j} w_{i j}^{\mathrm{CP}} r_{j}^{\mathrm{vis}}$. Given the activities of the action cells in $\mathrm{CP}$, the optimal movement according to the taxon strategy consists of an egocentric rotation by angle $\Psi^{\mathrm{CP}}$ defined as the preferred direction of the maximally active cell.

Action cells in nucleus accumbens (NA) are driven by the input from place cells, and their activity is described analogously to the cells in $\mathrm{CP}$, that is, $r_{i}^{\mathrm{NA}}=\sum_{j} w_{i j}^{\mathrm{NA}} r_{j}^{\mathrm{pc}}$, where $r_{j}^{\mathrm{pc}}$ is the activity of place cell $j$ (see Equation A9). The optimal action encoded by the activities of the cells in NA is a movement in an allocentric direction $\hat{\Psi}^{\mathrm{NA}}$ defined by the population vector

$$
\hat{\Psi}^{\mathrm{NA}}=\arctan \frac{\sum_{i} r_{i}^{\mathrm{NA}} \sin \left(\psi_{i}\right)}{\sum_{i} r_{i}^{\mathrm{NA}} \cos \left(\psi_{i}\right)} .
$$

Conversion of the allocentric angle $\hat{\Psi}^{\mathrm{NA}}$ to the egocentric motor action $\Psi^{\mathrm{NA}}$ is performed using an estimation of current heading $\Phi$ (see Equation A11) as $\Psi^{\mathrm{NA}}=\hat{\Psi}^{\mathrm{NA}}-\Phi$. Here, we apply a simple algorithmic approach for the readout of the action-cell activities and their conversion into a unified reference frame. In a more biologically plausible setting, both operations can be performed by using lateral interactions between actions cells (Deneve, Latham, \& Pouget, 1999, 2001).

How successful a strategy is on a particular trial is determined by the weights $w_{i j}^{\mathrm{CP}}$ for the taxon strategy and $w_{i j}^{\mathrm{NA}}$ for the locale strategy. We apply standard reinforcement learning theory (Sutton $\&$ Barto, 1998) to learn the weight values. The learning algorithms are identical for the CP and NA populations, and so, we omit the population index from the equations below. In our model, the value $Q$ of the movement in direction $\psi_{i}$ is given by the firing rate of the corresponding action cell, that is, $Q\left(s_{t}, a_{t}=\psi_{i}\right) \equiv r_{i}$, where $r_{i}$ is the activity of the $i$ th action cell. According to reinforcement learning theory, optimal action values on subsequent time steps should be related as $Q\left(s_{t}, a_{t}\right)=R_{t}+\gamma Q\left(s_{t+1}, a_{t+1}\right)$. The weights are adjusted on each time step so as to enforce this relationship:

$$
\Delta w_{i j}(t)=\beta \times \delta(t) \times e_{i j}(t),
$$

where $\beta=0.0001$ is the learning rate, $\delta(t)=R_{t}+\gamma Q\left(s_{t}, a_{t}\right)-$ $Q\left(s_{t-1}, a_{t-1}\right)$ is the reward prediction error, and $e_{i j}(t)$ is the eligibility trace that represents the memory of past actions. The eligibility trace of a synapse (Sutton \& Barto, 1998) is increased each time the synapse has participated in generating a movement and decays with a constant $\gamma \lambda$

$$
e_{i j}(t+1)=\exp \left[-\left(\psi_{i}-\Psi\right)^{2} / 2 \sigma_{\psi}^{2}\right] r_{j}+\gamma \lambda e_{i j}(t),
$$

where $r_{j} \equiv r_{j}^{\mathrm{pc}}$ for the locale strategy, and $r_{j} \equiv r_{j}^{\mathrm{vis}}$ for the taxon strategy. The exponential term ensures that actions $\Psi_{i}$ similar to the actually performed action $\Psi$ are also eligible for learning, thereby providing generalization in the action space (Strösslin, Sheynikhovich, Chavarriaga, \& Gerstner, 2005). A taxon trial is finished after a single orientation step, and so, the last term in Equation A5 is always zero for the taxon strategy (i.e., only one previous action is taken into account). To explore potentially useful actions, an $\varepsilon$-greedy action selection mechanism is used during learning: The optimal action is performed with probability $1-\varepsilon$, while a movement in a random direction is chosen with probability $\varepsilon(\varepsilon=0.1)$. 
Table A1

Model Parameters

\begin{tabular}{|c|c|c|}
\hline Parameter & Variable & Value \\
\hline 1. Time step, $\mathrm{s}$ & $\Delta t$ & 0.125 \\
\hline 2. Running speed of the simulated rat, $\mathrm{cm} / \mathrm{s}$ & $v$ & 16 \\
\hline 3. Horizontal view field of the simulated rats, degrees & $V$ & 300 \\
\hline 4. Size of the visual filter grid & $K$ & 9,216 \\
\hline 5. Gabor filter spatial width, degrees & $\sigma_{g}$ & 1.8 \\
\hline 6. Size of the action-cell populations & $N_{\mathrm{ac}}^{g}$ & 360 \\
\hline 7. Reward-based learning rate & $\beta$ & $10^{-4}$ \\
\hline 8. Future discount factor & $\gamma$ & 0.8 \\
\hline 9. Eligibility trace decay factor & $\dot{\lambda}$ & 0.8 \\
\hline 10. Probability of a random action & $\varepsilon$ & 0.1 \\
\hline 11. Width of generalization profile in the action space, degrees & $\sigma_{\psi}$ & 20 \\
\hline 12. Number of grid-cell populations & $N$ & 6 \\
\hline 13. Size of the grid-cell population & $N_{\mathrm{gc}}^{2}$ & 625 \\
\hline $\begin{array}{l}\text { 14. Standard deviation of the Gaussian noise in the self-motion } \\
\text { estimate of speed and direction, in percent of the change from }\end{array}$ & & \\
\hline previous step (testing/exploration) & $\sigma_{\eta}, \sigma_{\zeta}$ & $10 / 0.0$ \\
\hline 15. Lateral spread of the weights ${ }^{\mathrm{a}}$ & $\sigma_{\text {hex }}$ & 1.2 \\
\hline 16. Divisive normalization constant ${ }^{\mathrm{a}}$ & $\mu$ & 0.015 \\
\hline 17. Place-cell activity threshold & $\theta^{\mathrm{pc}}$ & 0.6 \\
\hline 18. Firing rate threshold to consider a cell highly active & $\theta^{\text {act }}$ & 0.7 \\
\hline 19. Number of active cells to consider a location as familiar & $T$ & 15 \\
\hline 20. Calibration constant for head direction (during testing/upon entry) & $\alpha_{\mathrm{hd}}$ & $0.7 / 1.0$ \\
\hline 21. View-cell directionality & $\sigma_{\Phi}$ & 1.2 \\
\hline 22. View-cell activity amplitude & $A$ & 1 \\
\hline 23. Width of the visual Gaussian in the filter space & $\sigma_{\mathrm{vc}}$ & 0.6 \\
\hline 24. Calibration constant for position (during testing/upon entry) & $\alpha_{\text {pos }}$ & $0.1 / 1.0$ \\
\hline
\end{tabular}

Note. The main free parameters that were adjusted to produce the simulation results are 5,11, 17, 18, and $20-24$. Other parameters were set either directly from available experimental data (e.g., 2 and 3; see text) or according to standard computational principles that govern learning (e.g., 7-10) or self-organization (e.g., 15 and 16).

${ }^{a}$ This parameter of the grid-cell network model is described in the supplementary materials.

\section{Grid Cells}

In our model, path integration is performed in a network consisting of $N$ identical grid-cell populations, which can be represented as two-dimensional sheets of recurrently connected neurons (Fuhs \& Touretzky, 2006; McNaughton et al., 2006). The recurrent connectivity in population $n=1, \ldots, N$ is chosen to form a two-dimensional attractor map, such that the shape of the activity profile at the stable state of recurrent dynamics is approximately constant, while its position $\vec{P}_{n}(t)$ on the sheet can change as a result of an external input. The position of the activity profile in the sheet is controlled by the animal's speed and direction of movement, as well as by visual input, as described below.

The architecture of the attractor network in each sheet, giving rise to the periodic triangular grid of Gaussian-like firing fields, is standard and is described in the online supplementary materials.

In this section, we describe the update of an activity profile position due to a pure self-motion input (i.e., path integration), while the correction of path integration by visual input is described later (see Equation A14). If the only available information about the movement comes from self-motion, then the position of the activity profile in the grid-cell population $n$ at time $t$ with respect to its position $\vec{P}_{n}(t-1)$ at the previous time step is given by

$$
\vec{P}_{n}^{\mathrm{pi}}(t)=\vec{P}_{n}(t-1)+\mathbf{R}_{n} \cdot\left(v_{n} \vec{s}\right),
$$

where $\mathbf{R}_{n}$ is the rotation matrix that defines the mapping of the movement direction of the animal to the movement direction of the activity profile across the sheet

$$
\mathbf{R}_{n}=\left\|\begin{array}{cc}
\cos \left(\xi_{n}\right) & -\sin \left(\xi_{n}\right) \\
\sin \left(\xi_{n}\right) & \cos \left(\xi_{n}\right)
\end{array}\right\|
$$

$v_{n}$ defines the mapping of the animal velocity to the velocity of the activity profile, and $\vec{s}=\left[\mathrm{v}^{\mathrm{pi}} \Delta t \cdot \cos \left(\Phi^{\mathrm{pi}}(t)\right), \mathrm{v}^{\mathrm{pi}} \Delta t \cdot \sin \left(\Phi^{\mathrm{pi}}(t)\right)\right]$ is the internal estimation of the change in position with speed and head direction given by

$$
\begin{gathered}
v^{\mathrm{pi}}(t)=v+\eta, \\
\Phi^{\mathrm{pi}}(t)=\Phi(t-1)+\Delta \Phi+\zeta .
\end{gathered}
$$

Here, $\eta$ and $\zeta$ are zero-mean normal random variables with standard deviations $\sigma_{\eta}$ and $\sigma_{\zeta}$ (see Table A1), that describe noise in the internal estimation of the constant animal velocity $v$ and rotation from the previous time step $\Delta \Phi$, respectively. The second line in Equation A8 describes an algorithmic implementation of the integration of head angular velocity over time, which is thought to be performed in the brain by a network of head direction cells (Ranck, 1984; Taube, Muller, \& Ranck, 1990a, 1990b). For neural models of the head direction network, see Arleo and Gerstner (2001) and Skaggs, Knierim, Kudrimoti, and McNaughton (1995). The correction of the pure self-motion estimate of the head direction at time $t$ is described later (see Equation A11).

In Equation $\mathrm{A} 7, \xi_{n}=(n-1) \times 15^{\circ} / N, n=1, \ldots, N$ defines relative orientations of different grids that, according to the experimental data from Barry et al. (2007), are distributed in the range from $0^{\circ}$ to $15^{\circ}$. The experimentally observed spacings between the grid vertices (Hafting et al., 2005) were simulated by appropriately 
tuning the values of the parameters $v_{n}$. Although grid orientations in the dorsomedial entorhinal cortex change independently of grid spacings, in the model we use the same index $n$ for both the spacings and orientations (an arbitrary permutation of $\xi_{n}$ independent of $v_{n}$ does not change any of the results). Upon the entry into a environment (i.e., at time $t=0$ ), the activity packets are assigned arbitrary positions in the corresponding charts and current heading is initialized by an arbitrary angle (we use $\Phi(0)=0$ ).

\section{Place Cells}

Place cells are driven by feed-forward input from the grid cells. Activity of cell $i$ is given by

$$
r_{i}^{\mathrm{pc}}=\left[\sum_{j} w_{i j}^{\mathrm{pc}} r_{j}^{\mathrm{gc}}-\theta^{\mathrm{pc}}\right]^{+},
$$

where $r_{j}^{\mathrm{gc}}$ is the activity of grid cell $j, w_{i j}^{\mathrm{pc}}$ is the connection weight, and $\theta^{\mathrm{pc}}$ is the activity threshold $\left([x]^{+}=x\right.$ if $x>0$ and $[x]^{+}=0$ otherwise).

During exploration, a place cell is recruited from a pool of cells if the current location is represented by less than $T=$ 15 sufficiently active place cells, that is, $\sum_{k} H\left(r_{k}^{\mathrm{pc}}-\theta^{\mathrm{act}}\right)$ $\leq T$, where $H(x)=1$ if $x>0$ and $H(x)=0$ otherwise. At the moment of recruitment, the weights $w_{i j}^{\mathrm{pc}}$ of the cell $i$ are set equal to the normalized activity of the grid cells, that is, $w_{i j}^{\mathrm{pc}}=r_{j}^{\mathrm{gc}}(t) / \sum_{j}\left(r_{j}^{\mathrm{gc}}(t)\right)^{2}$, where $t$ is the time step of exploration at the moment of recruitment. The weights of this form can be learned online using any self-normalizing competitive learning rule (see, e.g., Oja, 1982).

\section{Snapshot-Based Estimation of Head Direction}

Equation A8 represents a purely idiothetic update of the current estimate of head direction, and hence, (a) it is subject to the cumulative error, and (b) it has to be reset upon the entry to a familiar environment. The solution for both problems requires the knowledge of an allocentric estimate of the head direction. We use the following snapshot-based reorientation procedure. Suppose that a local view $i$ taken from a location $x$ has been stored in memory together with the corresponding head direction $\Phi_{i}$. At a later time, the model animal returns to the same location but with an unknown head orientation. To estimate the unknown head direction $\Phi$, we determine the angle $\Delta \Phi=\Phi-\Phi_{i}$ that leads to the best alignment of the current local view with the stored one. The goodness of an alignment with shift $\Delta \Phi$ is given by the cross-correlation $C_{i}(\Delta \Phi)$ between the current view and the stored view $i$. Searching for the maximum of $C_{i}$ across all possible shifts $\Delta \Phi$ yields the correct angle $\Phi=\Phi_{i}+\operatorname{argmax}_{\Delta \Phi}\left(C_{i}(\Delta \Phi)\right)$. Generalizing this idea to all the views taken from all different locations yields the allocentric head direction estimate

$$
\Phi^{\mathrm{vis}}=\operatorname{argmax}_{\Phi}\left(\left.\sum_{i} C_{i}(\Delta \Phi)\right|_{\Phi_{i}+\Delta \Phi=\Phi}\right) .
$$

Correction of the idiothetic estimate of head direction $\Phi^{\mathrm{pi}}$ (see Equation A8) is performed at each time step according to the following formula:

$$
\Phi(t)=\Phi^{\mathrm{pi}}+\alpha_{\mathrm{hd}}\left(\Phi^{\mathrm{vis}}-\Phi^{\mathrm{pi}}\right),
$$

where $\alpha_{\text {hd }}$ defines the amount of correction. Upon the entry to a familiar environment, head direction $\Phi$ is initialized with value $\Phi^{\text {vis }}$ by setting $\alpha_{\text {hd }}=1$. While our model of the head direction network (see Equations A8 and A11) is algorithmic (Franz, Schölkopf, Mallot, \& Bülthoff, 1998), rather than neuronal, it captures the fact that head direction cells are anchored to visual cues of the environment (Mizumori \& Williams, 1993).

\section{Readjustment of Path Integration}

View cells are used in the model to perform vision-based correction of the idiothetic estimate of the current position, performed in the network of grid cells (see Equation A6). Similarly to the simulated place cells, a new view cell is recruited at each time step during exploration, unless $T=15$ view cells are strongly active. Upon recruitment, the new view cell $i$ is initialized with a basis function center $\vec{\rho}_{i}^{\mathrm{vc}}$ with components $\rho_{i j}^{\mathrm{vc}}=r_{j}^{\mathrm{vis}}(t)$ that represents the current view at time $t$ (see Equation A2). At the same time, we store the estimated momentary head direction $\Phi_{i}=$ $\Phi(t)$ in which this view was taken (given by Equation A11). If the simulated rat observes later a different view with an estimated head direction $\Phi$, the stored view $\vec{\rho}_{i}^{\text {vc }}$ is rotated by the angular difference $\left(\Phi-\Phi_{i}\right)$, and view cell $i$ responds with the activity

$$
\begin{aligned}
r_{i}^{\mathrm{vc}}=A \exp \left(-\frac{1}{2 \sigma_{\mathrm{vc}}^{2}}\left[\frac{1}{\Omega_{i}}\left\|\vec{r}^{\mathrm{vis}}-\vec{\rho}_{\hat{\imath}}^{\mathrm{vc}}\right\|\right]^{2}\right) \\
\cdot \exp \left(\frac{\cos \left(\Phi-\Phi_{i}\right)-1}{\sigma_{\Phi}^{2}}\right),
\end{aligned}
$$

where $A$ is the amplitude, $\vec{\rho}_{\hat{i}}^{\text {vc }}$ is the center of the radial basis function after rotation by an amount $\Phi-\Phi_{i}$ (note the hat over the $i), \vec{r}^{\mathrm{vis}}$ is the vector of amplitudes of the Gabor wavelets corresponding to the currently observed view, II.II is the Euclidean norm, and $\Omega_{i}=V-\left(\Phi-\Phi_{i}\right)$ is a normalization factor that accounts for the overlap of the two visual fields in the angular plane. The second exponential term gives more weight to the comparisons with larger overlap $\Omega$ of the visual fields $\left(\sigma_{\Phi}=1.2\right)$. This factor ensures that only views that have been taken in similar head directions are compared with each other. Note that a simple view-matching approach without rotation and alignment of views would show negligible similarity for differences in head directions 
$\Phi-\Phi_{i}$ larger than the width of a single Gabor filter (i.e., $\sigma_{g}$ in Equation A1 expressed in angular coordinates). The value of the parameter $\sigma_{\mathrm{vc}}$, controlling the sensitivity of the visual system, was chosen such that the average width of the receptive field of a view cell was equal to $\approx 10 \mathrm{~cm}$. The view-cell activities given by Equation A12 represent a distributed code for the allocentric position of the simulated animal in a familiar environment. An example of the receptive field of a view cell and dependence of the view cell firing from the head direction of movement through the field (i.e., the head direction) are shown in Figure $3 \mathrm{C}$ in the main text.

Readjustment of the path-integration network is performed via associative connections between view cells and grid cells. We set the connection weight $w_{i j}^{\text {vis }}$ projecting from view cell $j$ to grid cell $i$ depending on the size of the spatial overlap between the regions in space where these cells are strongly active (i.e., the firing rate of the cell exceeds $\theta^{\text {act }}$ ), resulting in the following expression for the weight values:

$$
w_{i j}^{\mathrm{vis}}=Z^{-1} \sum_{x, y, \phi}\left[r_{i}^{\mathrm{gc}}(x, y, \phi)-\theta^{\mathrm{act}}\right]^{+} \cdot\left[r_{j}^{\mathrm{vc}}(x, y, \phi)-\theta^{\mathrm{act}}\right]^{+},
$$

where $Z$ is the normalization term ensuring that $\sum_{j}\left(w_{i j}^{\text {vis }}\right)^{2}=1, \forall_{i} ; x$, $y, \phi$ are the spatial positions and orientations visited by the simulated animal during exploration. Equation A13 can be interpreted as the result of Hebbian learning between view cells and grid cells, with $r_{j}$ being the presynaptic and $r_{i}$ the postsynaptic firing rates. Given the weights, a stimulation of the visual system alone will cause a location signal $\vec{P}_{n}^{\text {vc }}$ in each of the $N$ grid-cell populations, which is used to update the path integrator

$$
\vec{P}_{n}(t)=\vec{P}_{n}^{\mathrm{pi}}(t)+\alpha_{\mathrm{pos}}\left(\vec{P}_{n}^{\mathrm{vc}}(t)-\vec{P}_{n}^{\mathrm{pi}}(t)\right),
$$

where $\vec{P}_{n}^{\text {pi }}$ is the estimation of the new position due to the pure self-motion input (see Equation A6) and $\alpha_{\text {pos }}$ controls the importance of visual input. A relatively high value of the activity threshold for the weight values $\left(\theta^{\text {act }}=0.7\right)$ ensures that only strongly active grid cells and view cells become connected, such that the location signal induced by the visual system activates only a small subset of grid cells in each population.

If an animal enters a familiar environment (i.e., with existing place-cell population), the reorientation procedure is performed, which consists of (a) determining the allocentric head direction by calculating $\Phi^{\text {vis }}$ according to Equation A10, (b) determining the allocentric position by calculating view-cell activities $r_{i}^{\mathrm{vc}}$ according to Equation A12, and (c) initializing the activity profile positions in all grid-cell populations according to the view-cell activities propagated via the connections with strengths $w_{i j}^{\text {vis }}$.

Received February 6, 2008

Revision received March 5, 2009

Accepted March 5, 2009 